\title{
ARTICLE
}

\section{SF3B1 mutant MDS-initiating cells may arise from the haematopoietic stem cell compartment}

Syed A. Mian ${ }^{1, \star}$, Kevin Rouault-Pierre ${ }^{2, \star}$, Alexander E. Smith ${ }^{1,3}$, Thomas Seidl $^{1}$, Irene Pizzitola ${ }^{2}$, Aytug Kizilors $^{3}$, Austin G. Kulasekararaj, ${ }^{1,3}$, Dominique Bonnet $^{2} \&$ Ghulam J. Mufti,3

Despite the recent evidence of the existence of myelodysplastic syndrome (MDS) stem cells in 5q-MDS patients, it is unclear whether haematopoietic stem cells (HSCs) could also be the initiating cells in other MDS subgroups. Here we demonstrate that SF3B1 mutation(s) in our cohort of MDS patients with ring sideroblasts can arise from $\mathrm{CD}_{3}{ }^{+} \mathrm{CD}_{3}{ }^{-} \mathrm{CD}_{45 \mathrm{RA}}{ }^{-}$ $\mathrm{CD} 90^{+} \mathrm{CD}_{49 \mathrm{f}}{ }^{+} \mathrm{HSCs}$ and is an initiating event in disease pathogenesis. Xenotransplantation of SF3B1 mutant HSCs leads to persistent long-term engraftment restricted to myeloid lineage. Moreover, genetically diverse evolving subclones of mutant SF3B1 exist in mice, indicating a branching multi-clonal as well as ancestral evolutionary paradigm. Subclonal evolution in mice is also seen in the clinical evolution in patients. Sequential sample analysis shows clonal evolution and selection of the malignant driving clone leading to AML transformation. In conclusion, our data show SF3B1 mutations can propagate from HSCs to myeloid progeny, therefore providing a therapeutic target.

\footnotetext{
${ }^{1}$ Department of Haematological Medicine, King's College London School of Medicine, London SE5 9NU, UK. ${ }^{2}$ Human Normal and Malignant Haematopoiesis Stem Cells and Their Microenvironment Laboratory, The Francis Crick Institute, Lincoln's Inn Fields Laboratories, London WC2A 3LY, UK. ${ }^{3}$ Department of Haematology, King's College Hospital, London SE5 9RS, UK. * These authors contributed equally to this work.. Correspondence and requests for materials should be addressed to D.B. (email: dominique.bonnet@crick.ac.uk) or to G.J.M. (email: ghulam.mufti@kcl.ac.uk).
} 
$\mathrm{M}$ yelodysplastic syndromes (MDS) are clonal haematopoietic disorders with diverse phenotypes, characterized by varying severity of ineffective haematopoiesis, bone marrow (BM) dysplasia, variable rates of progression to acute myeloid leukaemia (AML), overall survival and response to therapy ${ }^{1,2}$. Recent studies have implicated defects of premessenger RNA splicing gene SF3B1 in the pathogenesis of MDS patients with ring sideroblasts (MDS-RS). SF3B1 mutations are present in up to $80 \%$ of the MDS-RS patients $s^{3-5}$ and strongly correlate with the presence of ringed sideroblasts ${ }^{4-7}$. It is noteworthy that all the mutations reported thus far in SF3B1 gene are heterozygous ${ }^{3-5,8}$, and knockout homozygous mouse models are embryonically lethal ${ }^{9}$.

Over the years, it has been reported that self-renewing haematopoietic stem cells (HSCs) continuously acquire somatic aberrations, while most of them are passenger mutations, some 'potent mutations' can constitute a reservoir of pre-leukaemic stem cells ${ }^{10-12}$. The first study to report clonal spectrum at a single-cell level through multiplex fluorescence in situ hybridization (FISH) analysis was in childhood acute lymphoblastic leukaemia ${ }^{13}$. However, the recent developments of genomic technologies, stem cell isolation as well as xenotransplantation models has started to lead to a better understanding of the complex clonal architecture and mutational hierarchy of phenotypically and functionally defined 'malignant stem cells' in AML ${ }^{14}$. A recent study on del(5q) MDS patients provided the first evidence of the genetic evolution and phenotypic hierarchy in $\operatorname{del}(5 \mathrm{q})$ MDS before AML transformation ${ }^{15}$.

In MDS-RS patients, the landscape of somatic mutations has become increasingly well defined ${ }^{3-5,7,16}$. However, the specific step within the developmental schema at which a clone attains a particular genetic aberration necessary to emerge or re-emerge as a dominant clone remains unknown. For instance, we have previously shown that the sequential acquisition of oncogenic alterations (such as FLT3 and RUNX1) in SF3B1 mutant MDS-RS patients results in disease progression to $\mathrm{AML}^{4}$. However, the origin of SF3B1 mutations, the detailed clonal composition (single-cell level), evolution as well as the engraftment kinetics of the haematopoietic cells that carry the SF3B1 mutations remain unknown. Therefore, we hypothesized that $S F 3 B 1$ mutations play a central role in MDS-RS pathogenesis, can arise from the more immature HSCs and hence provide a genetic marker to study the clonal evolution from the MDS disease to leukaemic transformation.

Our data demonstrate that SF3B1 mutations in MDS-RS patients can originate in rare HSCs and precede other known genetic lesions. Using xenotransplantation assays, we show that SF3B1 mutant clone alone or in association with other lesions confer clonal growth advantage over 'normal' cohabitating cells in NOD/SCID/IL2r $\gamma^{-1-}$ (NSG) mice. In addition, the xenograft NSG model recapitulates the clonal changes occurring in patients' bone marrow (BM). Furthermore, the fact that SF3B1-mutated stem cells constitute a 'pre-leukaemic' reservoir for more destabilizing mutations to occur points to the need of further large in vivo studies to identify, monitor and develop effective therapeutic strategies to prevent further subclonal evolution, recurrence and disease progression observed in MDS-RS patients.

\section{Results}

SF3B1 mutations arise in HSC and persist in myeloid progeny. Whole-exome sequencing (WES) of CD34 ${ }^{+}$cells from a cohort of 12 MDS-RS (8 RARS, 1 RCMD-RS, 2 RARS-T and 1 tMDS; Supplementary Table 1) including 8 previously reported ${ }^{4}$ and 1 congenital sideroblastic anaemia patient, revealed acquired mutations in SF3B1 in 11/13 cases (Supplementary Tables 1 and 2, Supplementary Fig. 1). A constitutional ALAS2 (R425C) gene mutation ${ }^{17-19}$ was detected in the patient with congenital sideroblastic anaemia, but no other mutations including SF3B1 (Supplementary Table 2) were observed in this case. Previous published studies have reported that recurrent gene mutations such as TET2, DNMT3A and JAK2 coexist in patients with SF3B1 mutations at variable frequencies (Supplementary Table 3$)^{4,8,20,21}$. In our cohort of 12 MDS-RS patients, SF3B1/ DNMT3A, SF3B1/TET2, SF3B1/JAK2 coexisted in 6, 2 and 1 patient, respectively. Using WES data, we were able to use the mutant allele burden $(\mathrm{MAB})$ as a mean for predicting the hierarchy of the mutations. In $2 / 6$ cases where DNMT3A and SF3B1 mutations coexist, SF3B1 mutation was present as a dominant clone (Supplementary Fig. 1, MDS1 and -6). In 3/6 patients, SF3B1 and DNMT3A mutations had similar MABs (MDS2, -10 and -12). In only one patient, DNMT3A (MDS11) had a DNMT3A clone higher than SF3B1 (70 versus 60\%, respectively).

Nevertheless, for TET2, and JAK2, where MABs between SF3B1 and these mutations were similar, it was not possible to decipher which mutation was ancestral without further clonal analysis. Unfortunately, for MDS10, MDS11 and 12, no viable BM cells were available to further dissect the clonal composition.

Here, we used 5 out of 12 MDS-RS patients, one congenital sideroblastic anaemia and three haematologically normal controls for further in vitro and in vivo analysis (Supplementary Table 4). An additional MDS-RS patient $(n=1$; MDS14) with SF3B1 mutation $\mathrm{H} 662 \mathrm{Q}$ was also included in this study (Supplementary Table 1).

To identify the target cell within the haematopoietic compartment, which carries the SF3B1 mutation, HSCs, multipotent progenitors (MPPs), granulocyte macrophage progenitors (GMPs) and megakaryocyte erythrocyte progenitors (MEPs) were isolated from the BM of four patients (MDS1, MDS2, MDS4 and MDS6) harbouring SF3B1 mutation (Supplementary Fig. 2A,B; Supplementary Table 1). To date, HSC in MDS studies have been enriched with the following markers $\mathrm{Lin}^{-} \mathrm{CD} 34^{+}$ $\mathrm{CD} 38^{-} \mathrm{CD} 90^{+} \mathrm{CD}_{45 \mathrm{RA}^{-}}$(ref. 22). To increase the HSCs purity, CD49f (integrin alpha 6) was used along with $\mathrm{Lin}^{-} \mathrm{CD} 34^{+} \mathrm{CD} 38^{-} \mathrm{CD} 90^{+} \mathrm{CD} 45 \mathrm{RA}^{-}$, which allowed us to enrich our population in long-term $\mathrm{HSCs}^{23}$. We performed targeted mutational analysis on the fluorescence-activated cell sorting (FACS)-purified cells from all patients and showed that SF3B1 mutations originate in the rare HSC population (Fig. 1). SF3B1 mutations were detected in the MPPs, GMPs and MEPs fractions in all patients (Fig. 1). Notably, the MAB was largely maintained between the HSCs, MPPs and more differentiated myeloid progenitors.

WES data from three of the patients (MDS1, MDS2 and MDS4) identified additional patient-specific mutations in $\mathrm{CD}_{3}{ }^{+}$cells. Next, we sought to determine whether these patient-specific mutations originate from the same HSC malignant pool as SF3B1 or whether they occur downstream of the stem cell differentiation in MPPs, GMPs and MEPs. Mutational analysis demonstrated that the majority of the patient-specific mutations including clinically important myeloid-specific genes such as DNMT3A and JAK2 were present in the rare HSC population and in the mature myeloid progenitors, that is, MPPs, GMPs and MEPs (Fig. 2). We also observed in some cases that 'patient-specific mutations' are either gained or lost at the mature progenitor level. This could be due to the fact that these mutations are present as minor clones $(<10 \% \mathrm{MAB})$ and are as such diluted further. None of the gene mutations including SF3B1 were detected in the mesenchymal stromal cells cultured from the patient's BM samples (data not shown). Furthermore, we and 

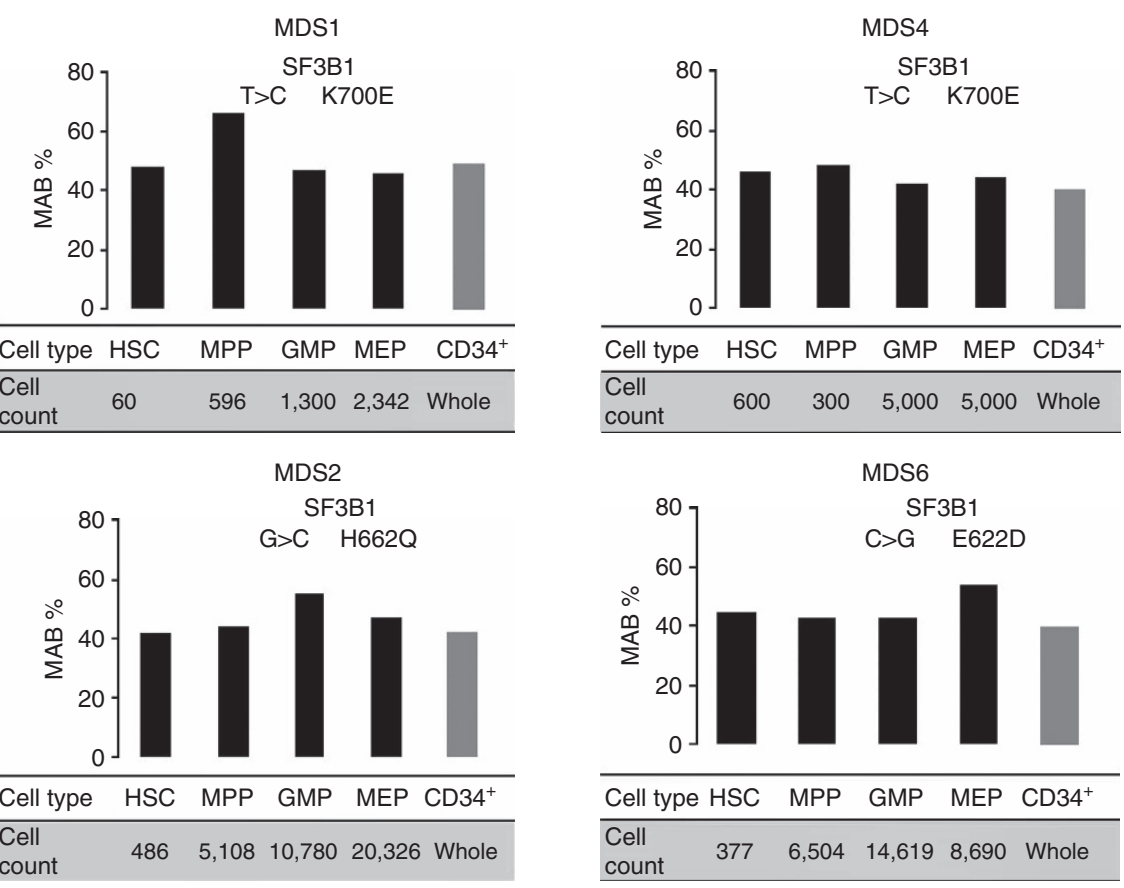

Figure 1 | SF3B1 mutations can propagate from HSCs to their progeny. SF3B1 mutations occurs in rare HSC phenotypic cells, is largely maintained in more committed progenitors MPPs as well as in more differentiated progenitors GMPs and MEPs. Tables show the progenitor cell counts (events detected by the FACS during cell sorting) for each patient sample. The average sequencing coverage across all amplicons was $\geq 800$ reads. Bar charts indicate the SF3B1 $M A B$ for the respective cell fraction. MAB for each cell population was confirmed by independent PCRs. MAB mutant allele burden ( $t$-test $P$ value $>0.05$ )

others have previously shown that SF3B1 mutations are not detected in T- or B cells in MDS-RS patients ${ }^{4,5}$. Taken together, in our cohort of patients SF3B1 mutations are clonally propagated by HSCs to their mature myeloid progenitors, but not to the lymphoid progenitors.

MDS-ICs are restricted to the rare HSC fraction. The NSG mice models in which primary human leukaemic cells are transplanted have previously been used to expand our understanding of the pathogenesis of human leukaemia ${ }^{13,24}$. Although MDS patient cells are difficult to engraft in xenograft models, the recent use of NSG mice as well as intra-bone injection (with or without coinjection of mesenchymal stroma cells) have been reported to yield higher engraftment ${ }^{15,22,25}$. Therefore, we prepared CD34 ${ }^{+}$ BM cells from four SF3B1-mutated MDS-RS patients (MDS1, TP1; MDS2, TP1; MDS3; MDS4) and one congenital sideroblastic anaemia patient (MDS5 with ALAS1 mutation), which was used as a control. Following on, these cells were then transplanted into NSG mice (Fig. 3a; Supplementary Table 4). In parallel, we injected adult $\mathrm{BM} \mathrm{CD} 34^{+}$cells from three healthy donors into NSG mice to control any lineage bias.

Engraftment levels based on human $\mathrm{CD} 45^{+}$cells harvested from engrafted mice at week 18-20 ranged between 0.1 and $4.9 \%$ (Fig. 3b; Supplementary Table 4). Majority of the cells engrafted in mice were of myeloid lineage with little or no lymphoid cell engraftment. In contrast, mice injected with $\mathrm{CD} 34^{+}$cells from a congenital sideroblastic anaemia patient showed myeloid as well as lymphoid cell engraftment (Fig. 3c; Supplementary Table 4). Similarly, engraftment observed in mice transplanted with $\mathrm{CD}_{3}{ }^{+}$cells from normal healthy controls exhibited a higher ratio of lymphoid/myeloid cell as compared with SF3B1 mutant cases (Fig. 3c). It is important to emphasize that B cells in MDSRS patients do not carry the SF3B1 mutation ${ }^{4}$. Therefore, data from our study suggest that the presence of $S F 3 B 1$ mutations, perhaps along with additional 'cooperating factors', adversely affects the lymphoid cell development in this model, which is in agreement with the previous xenotransplantation models proposed $^{15,22}$.

To confirm the nature of the human engrafted $\mathrm{CD} 45^{+} \mathrm{CD} 33^{+}$ cells (HECs) recovered from mice, mutational analysis was performed on HECs (Supplementary Table 5). Interestingly, the genotypic characteristics of patients' primary $\mathrm{CD} 34^{+} \mathrm{BM}$ cells were maintained in HEC, as demonstrated by the presence of SF3B1 mutations. As mentioned above, SF3B1 mutations are heterozygous ones, therefore a MAB of $50 \%$ implies that $100 \%$ of the cells carry the mutation. SF3B1 MAB remained largely unchanged between the primary $\mathrm{CD}^{+} 4^{+}$cells $(\approx 43 \%)$, and post-transplant HEC $(\approx 46 \%)$ in $3 / 4$ cases (Fig. $3 \mathrm{~d})$. This was irrespective of the type of SF3B1 mutations (K700E, $n=2$; $\mathrm{H} 662 \mathrm{Q}, n=2)$. In one RARS patient (MDS3), SF3B1 (K700E) $\mathrm{MAB}$ increased from $28 \%$ in primary CD34 ${ }^{+}$cells to $43 \%$ (average MAB between three mice) after xenograft transplantation. SF3B1 mutations were also observed in cells cultured in both CFC and long-term culture (LTC) conditions, at a variable MAB frequency (Fig. 2; Supplementary Fig. 5). Therefore, our data suggest a clonal advantage of the malignant mutant clone(s) over cohabitating normal HSCs in SF3B1 mutant MDS-RS cases.

Finally, we sought to determine which progenitor cells within the haematopoietic compartment were able to engraft in NSG mice. To address this, we isolated HSC, MPP, MLP, CMP and GMP from one MDS-RS patient (MDS14) with SF3B1 H662Q mutation and injected each fraction separately into mice. Interestingly, the mouse that received SF3B1-mutated HSCs engrafted and the SF3B1 H662Q MAB in mice was maintained (Table 1), which is similar to the recent data from Woll et al. ${ }^{15}$

Xenograft exhibits subclonal evolution of SF3B1 mutant clones. To further explore the clonal landscape and evolution of the SF3B1 mutant cells in our xenograft, we performed WES and/or targeted mutational analysis on the HECs. This strategy enabled 

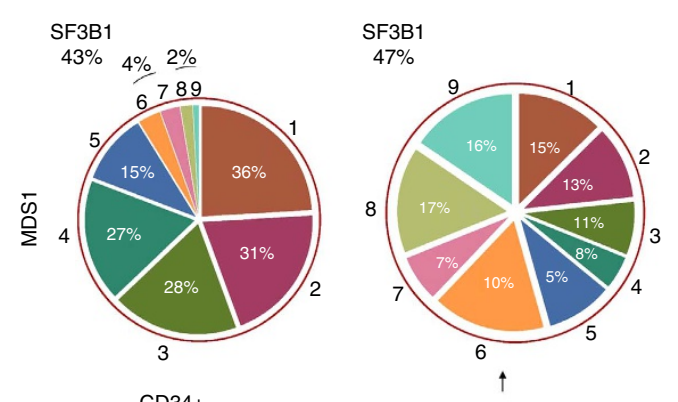

SF3B1
$40 \%$

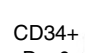

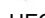
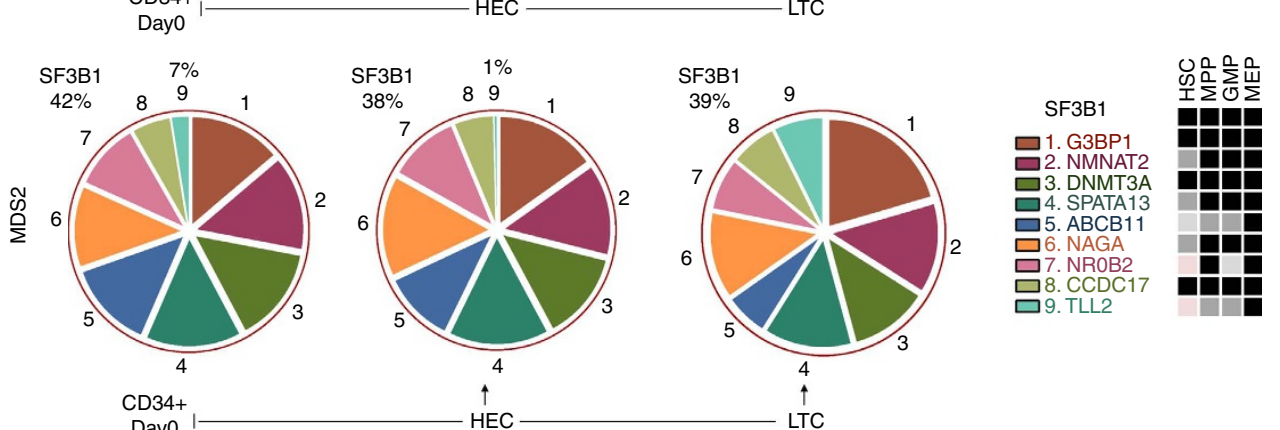
$\mathrm{SF} 3 \mathrm{~B} 1$
$28 \%$

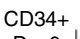
HEC
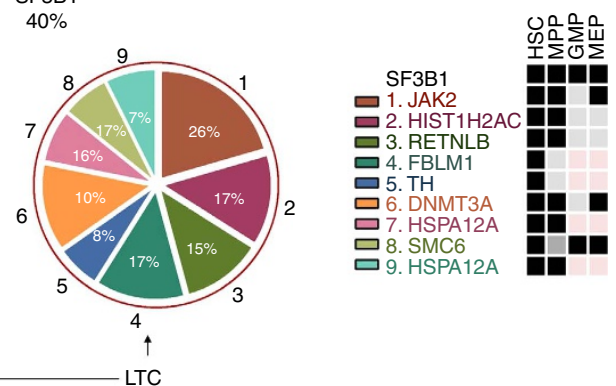

\section{SF3B1}

SF3B1
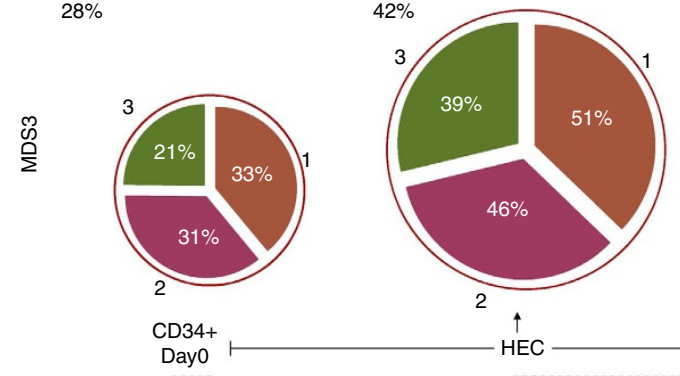

- HEC
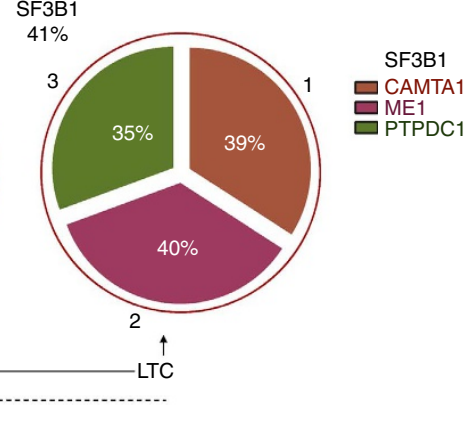

Figure 2 | Mutational architecture and subclonal evolution of SF3B1-mutated MDS-RS bone marrow cells. Panels show the gene mutations with their MABs in primary CD34+ bone marrow, HEC cells and LTC samples from each patient. Outer red circle represents the total SF3B1 clone and the inner sections of the circle represents the SF3B1 subclones. MABs for HEC samples are the average of all mouse samples (where applicable, that is, $>1$ mouse). Percentages displayed in the pie chart indicate the MAB frequency modulation in HEC and LTC samples versus $\mathrm{CD} 34{ }^{+}$day $0 .{ }^{\star} P<0.01 \%(t$-test) for difference in SF3B1 MAB frequency between $\mathrm{CD} 34^{+}$day 0 and HEC and/or LTC samples for the MDS3 sample. For more details about statistical analysis of the variation in MAB frequencies, refer to Supplementary Fig. 5. The table on the right side of each panel represents the mutation status of the respective genes in the HSC, MPP, GMP and MEP cell populations for each patient. Samples used to isolate cell fractions (HSCs, MPPs, GMPs and MEPs) were taken $\sim 18$ months later than the first sample used to isolate $\mathrm{CD}_{3}{ }^{+}$cells. Three independent PCRs were performed to confirm/determine the MAB throughout the experiments. In the tables on the right side, the black boxes indicate that MAB for gene mutations was the same as the primary $C D 34^{+}$cells, dark grey boxes show $M A B$ for gene mutations that was $\leq 2$-fold as compared to the primary $\mathrm{CD} 34^{+}$cells, light grey boxes represent $M A B$ for gene mutations that was $\geq 2$-fold as compared with the primary $\mathrm{CD} 34^{+}$cells and pink boxes indicate not detected or below background noise level. MAB mutant allele burden; HEC, human engrafted cell; LTC, long-term culture; BM, bone marrow; Mut, mutation.

us to reliably trace $S F 3 B 1$ and coexisting mutations as well as to detect de novo gene mutations. All the mutations were confirmed in at least one non-whole-genome amplified (WGA) HEC sample in all cases (see Methods for more details). The degree of quantitative accuracy of the allele burdens for WGA DNA was validated independently (Supplementary Fig. 3A,B; see Methods for details).

NSG xenograft maintained (or enhanced) the malignant SF3B1 clone in general; however, the subclonal population shift traced via the changes in the MABs was observed in 2/4 cases (Fig. 2; Supplementary Fig. 5; Supplementary Table 5). For example, in MDS1, JAK2 (V617F) MAB decreased from 36-15\% (average MAB for three mice; Fig. 2; Supplementary Fig. 5), whereas the $D N M T 3 A$ increased to $10 \%$. A similar trend was observed for other gene mutations in this patient. Interestingly, DNMT3A mutation was not initially detected in primary $\mathrm{CD} 34^{+}$cells by
WES. However, a follow-up high-depth targeted mutational analysis confirmed the presence of these mutations at a low level $(<5 \%)$ in the primary $\mathrm{CD}_{4}{ }^{+}$cells (Fig. 2; Supplementary Fig. 5). On the other hand, TLL2 mutation present in the MDS2 (7\% MAB) $\mathrm{CD} 34^{+}$cells almost disappeared in the NSG mice (1\% MAB, Fig. 2). In the rest of the cases, patient-specific mutations followed the same trend as SF3B1 clones, implying the presence of a single SF3B1 clone with multiple mutations. In all cases, similar clonal/subclonal changes were observed in pooled CFC and LTC assays.

Together our data demonstrate that $S F 3 B 1$ malignant cells were maintained or even enhanced in NSG mice and that subclonal fluctuation could be detected in certain cases. Of note, all the mutations present in the NSG mice were traced back to the patients' primary $\mathrm{CD} 34^{+}$cells and no new mutations were detected in the NSG mice. 
a

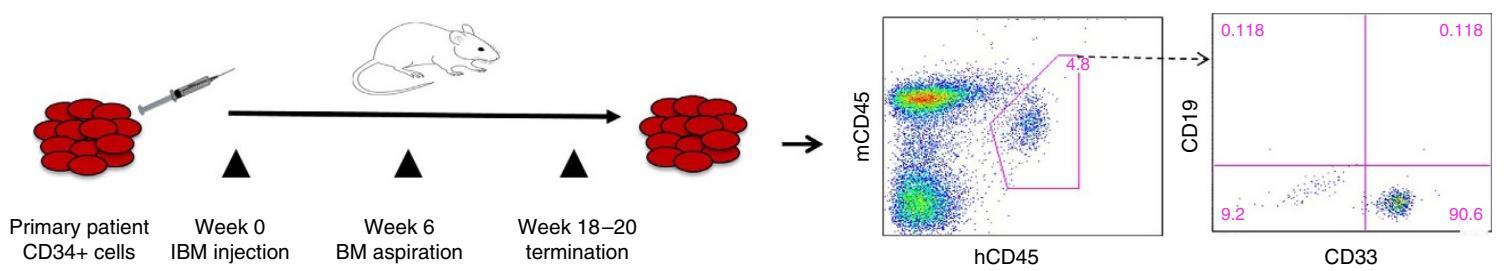

b
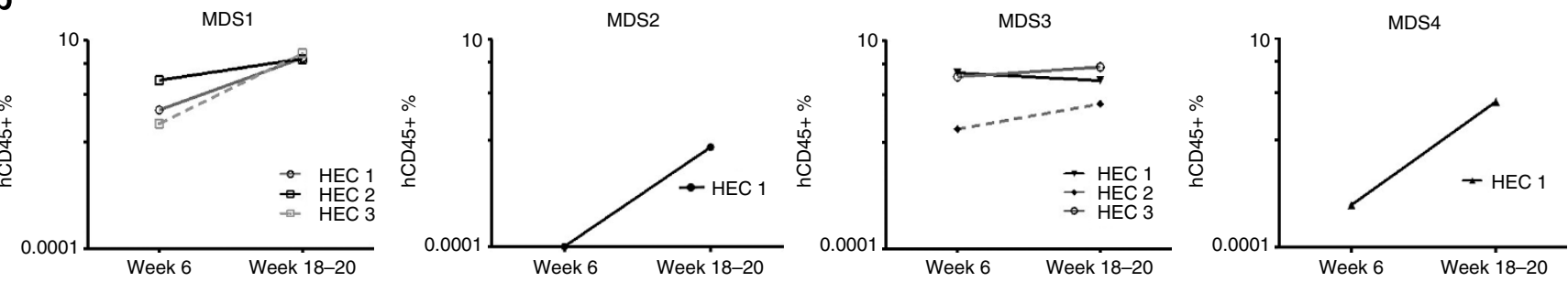

C
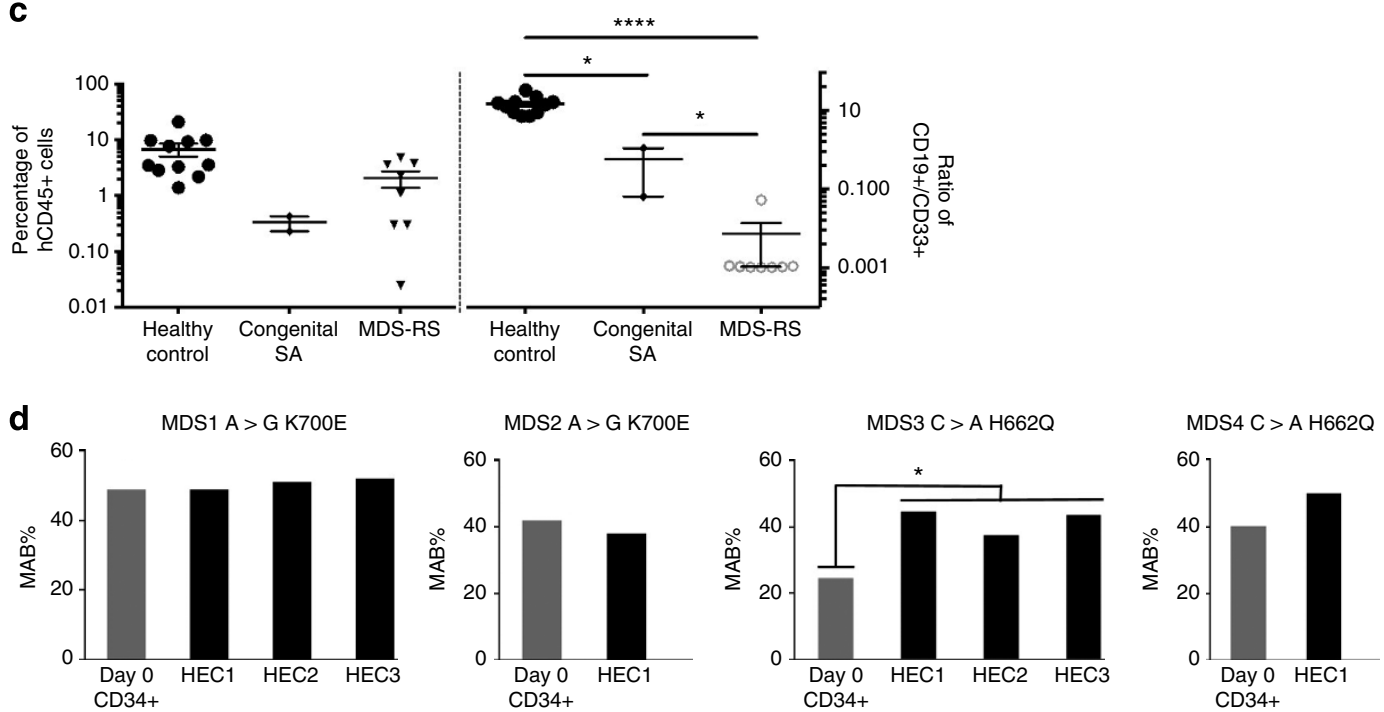

Figure 3 | Engraftment and genotype of human RARS-originated and control haematopoietic cells in the bone marrow of NOD/SCID/IL2r $\gamma^{-/-}$mice. (a) Schematic representation of the xenograft model. Right-hand side panel shows the flow cytometry profile of bone marrow cells recovered from one human-cell-engrafted mice. The majority of human CD45-expressing cells were positive for a myeloid marker CD33 ${ }^{+}$in all analysed cases in this study. (b) Percentage of human CD45 cells in the bone marrow of NSG mice at 6 and 18-20 weeks after transplantation (MDS1, $n=3 ;$ MDS2, $n=1 ;$ MDS3, $n=3$ and MDS4, $n=1$ ). (c) Percentages of human haematopoietic cells and ratio of CD19 ${ }^{+} / \mathrm{CD}_{3} 3^{+}$isolated from the bone marrow of mice engrafted with either MDS-RS ( $n=4$ patients and transplanted in a total eight mice) or healthy controls CD34 ${ }^{+}$cells $(n=3$ healthy donors and transplanted in a total of 11 mice) or congenital sideroblastic anaemia patient ( $n=1$ patient transplanted in two mice). The $y$ axis (left) represents the percentage of the human CD45 ${ }^{+}$cells present in the total mouse bone marrow. The $y$ axis (right) represents the ratio of the human $\mathrm{CD}_{19}{ }^{+}$versus human $\mathrm{CD} 33^{+}$cells within the human $\mathrm{CD} 45^{+}$cells recovered following xenotransplant. MDS xenografts showed a significant skewing towards myeloid lineage. ${ }^{\star} P<0.05,{ }^{\star \star \star \star} P<0.0001$ ( $t$-test). (d) Targeted mutational analysis shows the presence of concordant SF3B1 mutations in primary CD34 ${ }^{+}$bone marrow sample (grey) and xenograft (black) in all analysed cases. Three independent PCR/sequencing experiments were performed to confirm/determine the mutant allele burden throughout the experiments. The sequencing coverage across the SF3B1 amplicons was $\geq 1,000$ reads. ${ }^{\star} P<0.01$ ( $t$-test).

Clonal analysis reveals mutational architecture in MDS-RS. Previous studies have described mutational landscape of SF3B1mutated patient $\mathrm{BM}$ cells $^{3-5,8}$. However, these studies have inferred the clonal architecture in the total BM samples through the identification of clusters of coexisting mutations with similar MABs, and this does not necessarily recapitulate the actual subclonal architecture of the patients' BM compartment. Therefore, to gain insight into the clonal landscape of coexisting mutations in SF3B1 mutant MDS-RS patients, we performed a single-cell clonogenic assay using $\mathrm{CD} 34^{+}$cells from four patients (MDS1, time point 1; MDS2, TP1; MDS3; MDS4), followed by targeted mutational analysis of the patient-specific mutations.
Initial mutational analysis performed on individual CFC colonies from MDS1 (48-96 colonies), MDS2 (47 colonies), MDS3 (46 colonies) and MDS4 (30 colonies) using gene-specific primers targeting SF3B1 mutations revealed that $97 \%$, 98\%, 96\% and $77 \%$ of the colonies carried SF3B1 mutant gene, respectively (Fig. 4; Supplementary Fig. 6). Moreover, further analysis of the 'patient-specific' gene mutations in these cases revealed the complex heterogeneity within the SF3B1-mutated colonies. For example, MDS1 and MDS2 had a marked subclonal heterogeneity (Fig. 4). Notably, driver mutations such as DNMT3A were present in 44/47 MDS2 colonies, suggesting that it may have been acquired at a later time point during disease progression. MDS1 demonstrated six different $S F 3 B 1$ subclones where 15/48 colonies 
Table 1 | Engraftment of sorted haematopoietic cells in NSG.

\begin{tabular}{|c|c|c|c|c|c|c|c|}
\hline & $\begin{array}{l}\text { SF3B1 mutation } \\
\text { CD34 } \\
+ \text { cells }\end{array}$ & $\begin{array}{l}\text { Mouse } \\
\text { model }\end{array}$ & $\begin{array}{c}\text { Cells } \\
\text { injected }\end{array}$ & $\begin{array}{l}\text { Engrafted } \\
\text { mice }\end{array}$ & $\begin{array}{l}\text { hCD33\% cells } \\
\text { hCD19\% cells } \\
\text { (12 weeks) }\end{array}$ & $\begin{array}{l}\text { SF3B1MAB in } \\
\text { CD34 }{ }^{+} \text {cells } \\
\text { (day } 0)\end{array}$ & $\begin{array}{c}\text { SF3B1MAB in HEC } \\
\text { (12 weeks) }\end{array}$ \\
\hline \multicolumn{8}{|l|}{$\mathrm{Lin}^{-} \mathrm{CD} 45^{+} \mathrm{CD} 34^{+}{ }^{+} \mathrm{CD} 38^{-}$} \\
\hline $\begin{array}{l}\mathrm{HSC} \\
\mathrm{CD} 45 \mathrm{RA}^{-} \mathrm{CD} 90^{+} \mathrm{CD}_{49 f}{ }^{+}\end{array}$ & H662Q & NSG & 1,629 & $1 / 1$ & $\begin{array}{l}100 \% \\
0.00 \%\end{array}$ & $46 \%$ & $46 \%$ \\
\hline $\begin{array}{l}\text { MPP } \\
\text { CD45RA - CD90 - CD49f- }\end{array}$ & H662Q & NSG & 29,944 & $0 / 1$ & NA & NA & NA \\
\hline \multicolumn{8}{|l|}{$\mathrm{Lin}^{-} \mathrm{CD}^{-} 5^{+} \mathrm{CD} 34^{+} \mathrm{CD} 38$} \\
\hline $\begin{array}{l}\text { MLP } \\
\text { CD45RA }+ \text { CD90 }^{-}\end{array}$ & H662Q & NSG & 51 & $0 / 1$ & NA & NA & NA \\
\hline $\begin{array}{l}\text { CMP } \\
\text { CD45RA }-{ }^{-} \text {CD135+ }\end{array}$ & $\mathrm{H} 662 \mathrm{Q}$ & NSG & 16,231 & $0 / 1$ & NA & NA & NA \\
\hline $\begin{array}{l}\text { GMP } \\
\text { CD45RA }+{ }^{+} \text {CD135 }+\end{array}$ & $\mathrm{H} 662 \mathrm{Q}$ & NSG & 28,217 & $0 / 1$ & NA & NA & NA \\
\hline $\begin{array}{l}\text { FACS, fluorescence-activated cell sorting; } \\
\text { myelodysplastic syndrome; MDS-IC, MD } \\
\text { The table shows the engraftment of FACS-i } \\
\text { weeks, mice were killed and the bone marr }\end{array}$ & $\begin{array}{l}\text { MP, granulocyte macrop } \\
\text { tiating cell; MDS-RS, N } \\
\text { ted haematopoietic cell } \\
\text { was analysed. Only the }\end{array}$ & $\begin{array}{l}\text { progenito } \\
\text { patients } w \\
\text { ons injecte } \\
\text { HSC fracti }\end{array}$ & $\begin{array}{l}\text { EC, human } \\
\text { ring siderobl } \\
\text { to NSG mice. } \\
\text { gave rise to }\end{array}$ & $\begin{array}{l}\text { Igrafted CD45 } \\
\text { st; MPP, multipc } \\
\text { rogenitor cells } f \\
\text { myeloid-restricte }\end{array}$ & $\begin{array}{l}3^{+} \text {cell; HSC, haem } \\
\text { progenitor; NA, not } \\
\text { ne MDS-RS patient } w \\
\text { graftment, indicating } t\end{array}$ & $\begin{array}{l}\text { tic stem cell; MAB, } \\
\text { ble; NSG, NOD/SCIL } \\
\text { lated and injected sep } \\
\text { ure of the MDS-ICs ir }\end{array}$ & $\begin{array}{l}\text { ant allele burden; MDS, } \\
2 r \gamma-O D \text { mice. } \\
\text { tely into NSG mice. After } 12 \\
\text { s rare HSC fraction. }\end{array}$ \\
\hline
\end{tabular}

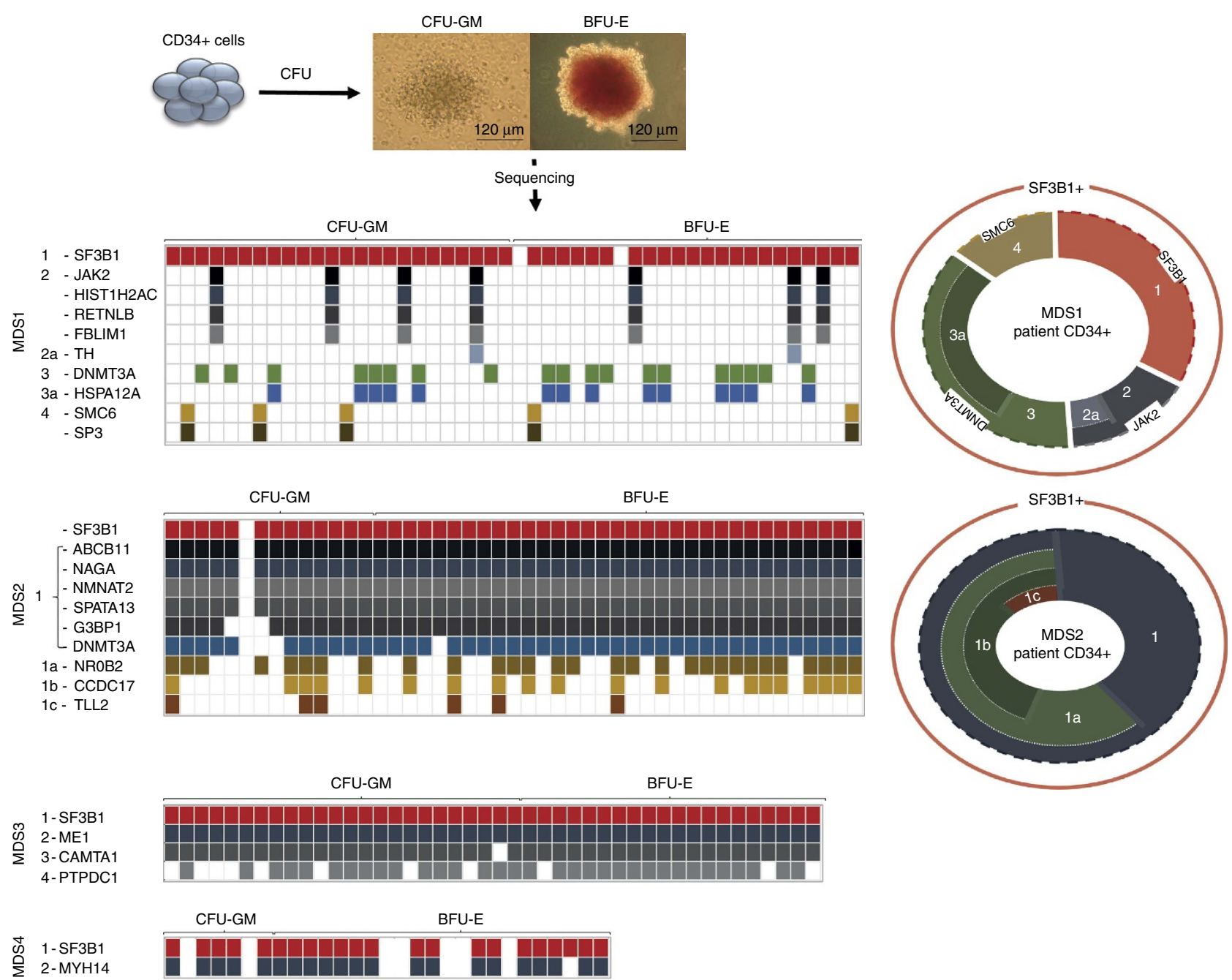

Figure 4 | SF3B1 mutations in single-cell colonies. Mutational analysis was performed on BFU-E and CFU-GM colonies derived from primary CD34 ${ }^{+}$ patient cells (MDS1, MDS2, MDS3 and MDS4). Each column represents an individual CFC colony. Circle (MDS1 and MDS2) on the right is the representative of the overall clonality observed in the clonogenic assays (left) in MDS1 and MDS2 and total CD34 ${ }^{+}$cells. The outer red circle represents the total SF3B1 clone, while the inner circle depicts subclones. WT, wild type. 
had SF3B1 mutations alone (Fig. 4; Supplementary Fig. 6). Interestingly, some of these subclones were mutually exclusive, especially subclones with known driver mutant genes such as $J A K 2$ and DNMT3A, therefore representing a branching multi-clonal evolution (Fig. 4). In contrast, the simplest of the genetic architectures was present in MDS3 and MDS4 where only two and three mutant SF3B1 subclones were observed, respectively (Fig. 4).

It is noteworthy that CFC individual colonies with wild-type SF3B1 (MDS1, $n=2$; MDS2, $n=1 ;$ MDS3, $n=2$ and MDS4, $n=7$ ) had no additional gene mutations among those screened (Fig. 4; Supplementary Fig. 6). We therefore provide further evidence that $S F 3 B 1$ mutations are present in the majority of the $\mathrm{CD}_{3}{ }^{+}$cells and, importantly, precede other driver mutations such as DNMT3A and JAK2 as evidenced here. This implies that SF3B1 mutation is the 'founder mutation' in these MDS-RS patients.

Xenograft recapitulates the clonal evolution in patients $\mathrm{BM}$. To ascertain whether the xenograft clonal changes observed, occur in the MDS-RS patient BM, we performed targeted mutational screening on sequential samples (total nucleated cells) obtained from two patients (MDS1, $\approx 18$ months after initial sampling with no clinical disease progression, TP2; MDS2, $\approx 29$ months after the first sampling at the AML transformation stage, TP2; Supplementary Table 1). SF3B1 mutations were largely unchanged between the two time points in both patients (Fig. 5;
Supplementary Fig. 7). However, in MDS1, a change in the MAB of additional somatic mutations was observed in the sequential sample (TP2; Supplementary Fig. 7). For example, JAK2 subclone (V617F, 36-13\%) diminished (Fig. 5), whereas DNMT3A clone increased from $4-15 \%$, in the sequential sample (TP2, Fig. 5). Strikingly, cells from TP1 engrafted into the mice and harvested at week 20, demonstrated similar subclonal changes as observed in the sequential patient TP2 (Fig. 5). These data along with our clonogenic data suggest that four $S F 3 B 1$ subclones exist in this patient; however, 'isolated SF3B1' and 'SF3B1 plus DNMT3A' clones have a growth advantage over 'SF3B1-JAK2' clone and others in NSG mice as well as in the human BM environment (TP2).

In MDS2, CCDC17 and TLL2 mutations were not detected in the sequential sample while the rest of the mutations were largely maintained. A similar trend was observed in the NSG mice where TLL2 gene mutation diminished to $1 \%$ in the HEC sample (Supplementary Fig. 7). Altogether, our data suggest that the xenograft model does recapitulate some of the clonal changes occurring in the patients' BM in one case, while as in another case where AML transformation occurred, a partial recapitulation was observed. This could be probably due to the stochastic acquisition of additional genomic lesion at the later stage of the disease. Meanwhile, it is noteworthy that the 'incubation period' of the MDS BM cells in NSG mice was only 20 weeks while the patient (MDS2) transformed to AML 29 months after the first MDS sampling.

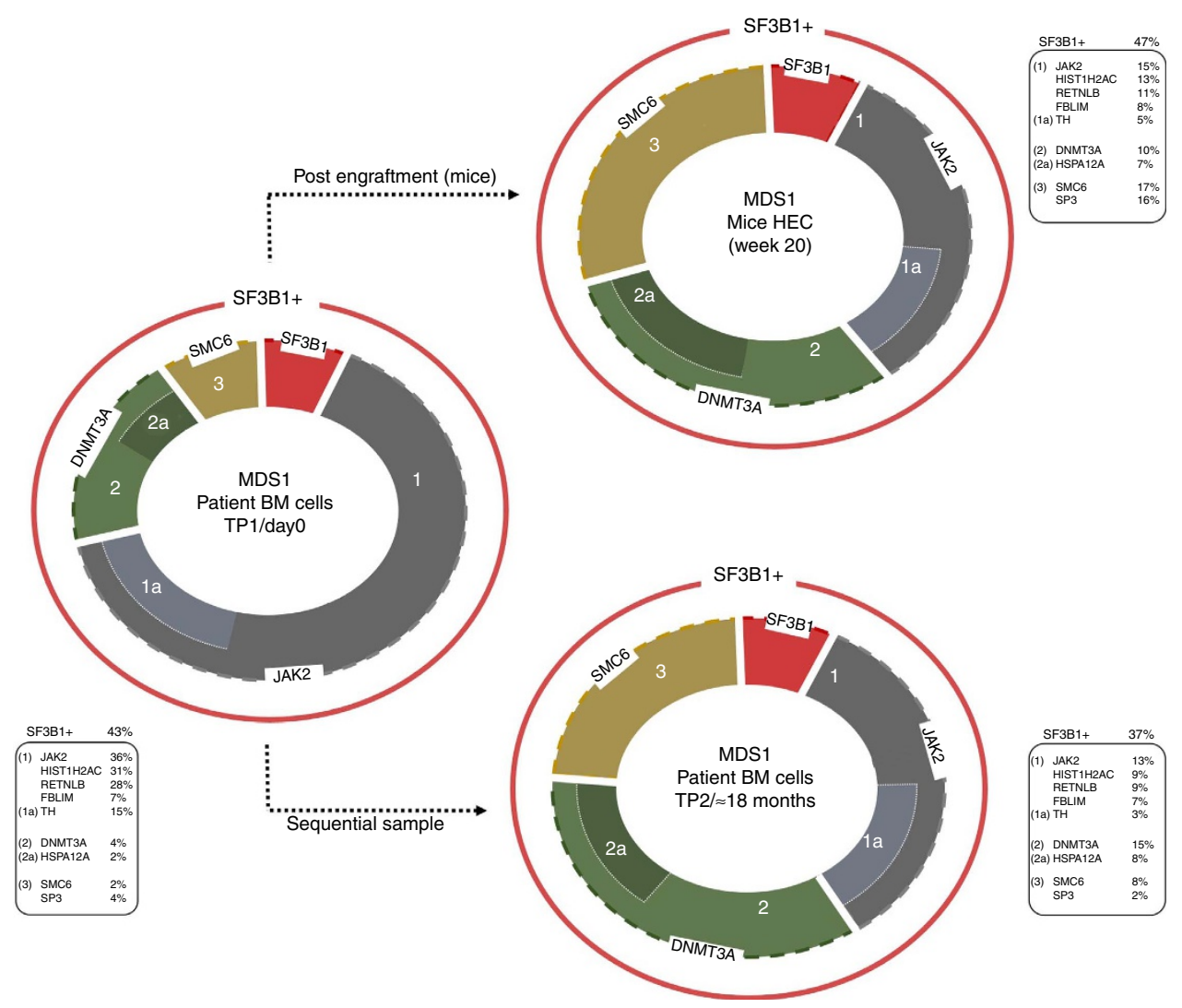

Figure 5 | Xenograft recapitulates the clonal changes occurring in RARS patient bone marrow compartment. Mutational analysis of sequential primary TNC (total nucleated cells) sample from one patient (MDS1). Primary patient sample was received at two different time points, MDS1 bone marrow cells (TP1, first sample) and MDS1 bone marrow cells (TP2, second sample). Mutational analysis was also performed on human engrafted cells, which were obtained from mice transplanted with the patient sample received at time point 1. The length of the arc represents the MABs. Gene MAB for human engrafted cells is the average between three animal experiments. Three independent PCR/sequencing experiments were performed to confirm/determine the mutant allele burden throughout the experiments. Error Bar in the bar chart were derived from the PCR/sequencing technical replicates. MAB, mutant allele burden; TP, time point; HEC, human engrafted cells; BM, bone marrow cells. 
Sequential lesions in SF3B1 clones leads to transformation. MDS2 patient evolved into AML 29 months after the first MDS sample was taken (MDS2, TP1). It is important to note that leukaemic transformation in RARS disease is a very rare event. Initially, we performed FACS analysis on BM samples (TP3) to examine the phenotypic changes that could have occurred in the BM leading to AML (Fig. 6a). The frequency of $\mathrm{CD} 34^{+}$cells in BM mononuclear cells (MNCs) at AML stage (at 29 months) was significantly higher as compared with the MDS stage 35.3 and $1.54 \%$, respectively. Interestingly, a remarkable shift of cell populations was observed within the $\mathrm{CD} 34^{+} \mathrm{CD} 38^{-}$and $\mathrm{CD} 34^{+} \mathrm{CD}^{+}{ }^{+}$compartments showing a marked increase in MLPs (>99\%) and GMPs (>99\%) at the AML stage (TP3) (Fig. 6a). If this result implies the switch from a stem cell disease to a progenitor disease, where a MDS progenitor would have acquired self-renewal ability and became AML blast, we should be able to identify MDS-initiating cells (MDS-ICs) still present at the AML stage. Thus, we sought to investigate the contribution of additional genomic lesions (chromosomal aberrations; gene mutations, known 'driver' or 'passenger' mutations) acquired at the AML stage. Metaphase cytogenetics at the AML stage revealed complex chromosomal changes (Supplementary Table 1) including $\operatorname{del}(7 \mathrm{q})$. To understand whether $\operatorname{del}(7 \mathrm{q})$ was a major event in the transformation process, we traced back this chromosomal aberration by FISH analysis (Supplementary Fig. 8A) of the whole BM sample, MLPs and GMPs at the AML stage and also on the whole BM sample at the MDS stage (TP1). Two chromosome-7 aberrations were detected at the AML stage of the disease with $93 \%$ harbouring del (7q36) and $7 \%$ containing del(7q22-7q36), in the total MNCs, in the MLPs and in the GMPs (Fig. 6b,c). Interestingly, only $\operatorname{del}(7 q 36)$ was traced back to the MDS stage of the disease; however, the frequency was significantly lower, that is, $2 \%$ (Fig. 6d,e). We were unable to perform FISH analysis on the phenotypically defined HSCs, MPPs, CMPs and MEPs due to the low frequency of these progenitors in the BM at the AML stage. It is noteworthy that FISH analysis on the LTC-derived cells (MDS stage $\mathrm{TP} 1$ ) showed low-level del(7q36) clone (3\%; Fig. 6d), therefore suggesting that the major clone containing del( $7 q 36)$ is an early lesion acquired in the SF3B1 mutant rare HSCs at an MDS stage of the disease. However, SNV profile through WES on LTC showed no change in the loss of heterozygosity at chromosome 7, and similar results were observed at the MDS stage for $\mathrm{CD}^{+} 4^{+}$cells, HEC (MDS stage) and $\mathrm{CD}^{+}$cells (Supplementary Fig. 8B).

As the $\operatorname{del}(7 \mathrm{q})$ does not seem to be a 'marker' of the AML transformation, we sought for other targets through WES. Results showed that SF3B1 and other additional gene mutations including DNMT3A were maintained; however, CCDC17 and TLL2 were not detected (Fig. 6f). Further analysis revealed additional patient-specific mutations, including SUV420H1 (L645F) novel mutation (Fig. 6f), which is a histone methyltransferase that specifically dimethylates 'Lys-20' of histone H4. Due to the functional importance of this gene and its known association with breast cancer $^{26,27}$, we followed this gene mutation and performed high-depth (sequencing depth $>10,000$ reads) targeted mutational screening from samples at the MDS stage (TP1 and TP2). Unlike $\operatorname{del}(7 \mathrm{q})$, this mutation was not detected in any of the previous MDS samples (Fig. 6f).

Furthermore, mutational analysis of the HSC, MLP and GMP compartments (AML stage) revealed that SF3B1 and DNMT3A mutations were present in all the compartments. However, SUV420H1 mutation was not detected in HSCs but originated from MLPs and propagated to GMPs, supporting the hypothesis of mutations acquired at a MDS progenitor stage conferring selfrenewal ability (Fig. 6g; Supplementary Fig. 8C). Taken together, our data suggest that SF3B1 mutation precedes $\operatorname{del}(7 \mathrm{q})$ aberration and is maintained throughout the disease evolution (Fig. 6h). Furthermore, acquisition of additional 'potent' mutations such as SUV420H1 in the 'SF3B1-del(7q)' subclone in the MLP compartment led to the expansion of this MLP clone and hence transformation to AML (Fig. 7). Overall, our results provide evidence for the existence of MDS-ICs in the MDS-RS disease, which persist during the AML transformation and could potentially provide a reservoir of the pre-leukaemic stem cell, as well as play an important role during disease relapse.

\section{Discussion}

Next-generation sequencing technologies have greatly improved our understanding of the genetic landscape in cancer. Previous work in AML and MDS demonstrated the existence of clonal heterogeneity that evolves upon disease progression and/or relapse ${ }^{14,28-31}$. To date, various studies including ours have shown that SF3B1 is the most frequently mutated gene in MDS with a remarkably strong association with a specific disease phenotype, that is, the presence of ring sideroblasts ${ }^{3-5}$, suggesting that this mutation is casually linked to event-defining ring sideroblastic anaemia. However, it is noteworthy that studies so far have not determined the origins of SF3B1 mutations within the haematopoietic compartment and the composition of the SF3B1 subclones. Similarly, the functional heterogeneity of genetically defined SF3B1 mutant subclones has not been delineated. SF3B1 mutations are detected in low-risk disease and therefore, these patients in general have low frequency of other gene mutations ${ }^{4,8,20}$. However, the recurrent gene mutations that coexist with SF3B1-mutated patients are TET2, DNMT3A and JAK2. In fact, based on previous data, TET2 (26\%) and DNMT3A (21\%) occur roughly at the same frequency in the SF3B1 mutant patients, whereas mutations such as ASXL1 coexist only in $8 \%$ of the SF3B1-mutated patients (Supplementary Table 3). Here we used WES, capture-based targeted highdepth sequencing, long-term culture assay, xenotransplantation as well as single-cell clonogenic assays to study the characteristics of SF3B1 mutant haematopoietic cells. Mutational analysis of the SF3B1 gene in haematopoietic stem/progenitor cells reveals that $S F 3 B 1$ mutations arising in phenotypically defined $\mathrm{CD} 34^{+} \mathrm{CD} 8^{-} \mathrm{CD} 45 \mathrm{RA}^{-} \mathrm{CD} 90^{+} \mathrm{CD}^{-} 9 \mathrm{f}^{+}$stem cells therefore unveil the MDS-ICs existence in RARS that propagate mutations to their myeloid progenitors, such as MPPs, GMPs and MEPs. This was also reflected in clonogenic assays where SF3B1 mutations were detected either alone or in combination with other mutations in individual CFC colonies, therefore implying that SF3B1 aberrations precede other gene mutations. Similar results have been described recently, in MDS patients with concurrent SF3B1 and $\operatorname{del}(5 \mathrm{q})$ aberrations, where SF3B1 mutations were shown to be present in $\mathrm{CD} 34^{+} \mathrm{CD} 38^{-} \mathrm{CD} 90^{+}$ stem cells in these patients ${ }^{15}$.

In our study, xenotransplantation of $\mathrm{CD} 34^{+} \mathrm{BM}$ from MDS samples in immunodeficient mice demonstrated the persistent long-term myeloid-restricted engraftment in the majority of cases. In contrast, NSG data from one congenital sideroblastic anaemia patient carrying an ALAS2 mutation and other healthy donors showed a multi-lineage engraftment with high a percentage of the lymphoid/myeloid cell ratio. It is noteworthy that all four patients in our study had only one common genomic aberration, that is, SF3B1. In addition, 2/4 patients (MDS2 and MDS3) had a lower mutation rate (2-4 mutations per exome), which is less as compared with that in AML $(\approx 13)$, chronic lymphocytic leukaemia $(\approx 12)$, and was significantly lower as compared with multiple myeloma $(\approx 33)$, hence representing a much more homogenous clonal population. 
a

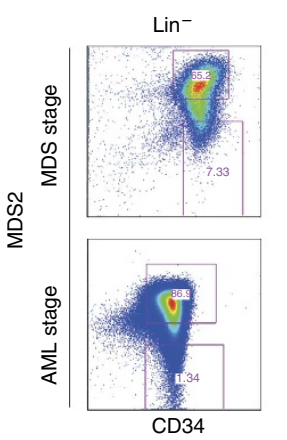

C
$\operatorname{Chr} 7$
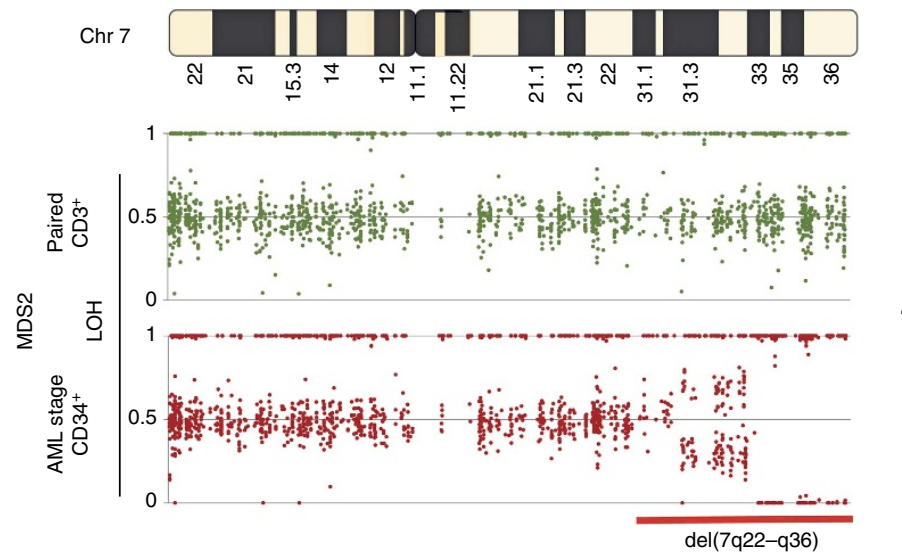

b

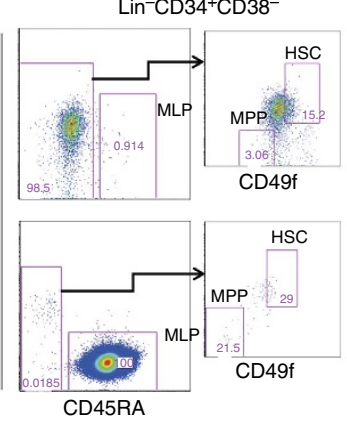

$\operatorname{Lin}^{-} \mathrm{CD} 34^{+} \mathrm{CD} 38^{+}$
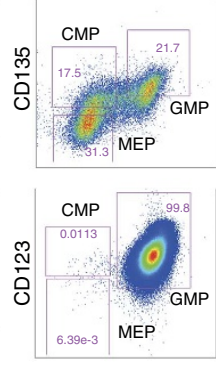

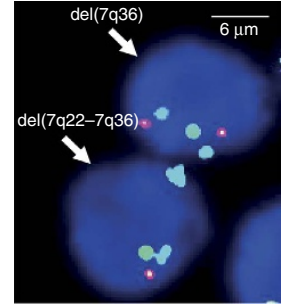

d
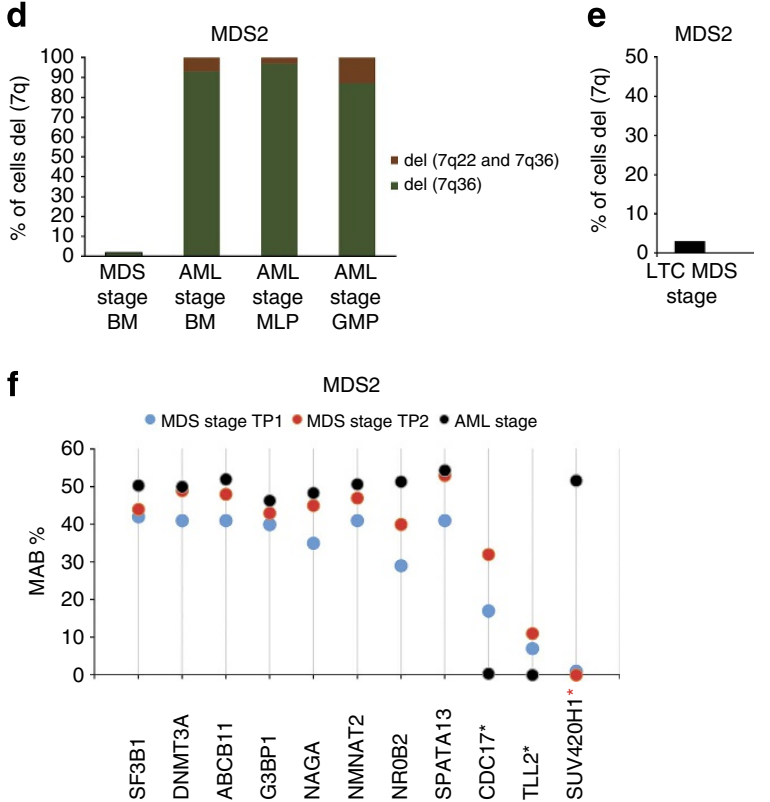

g

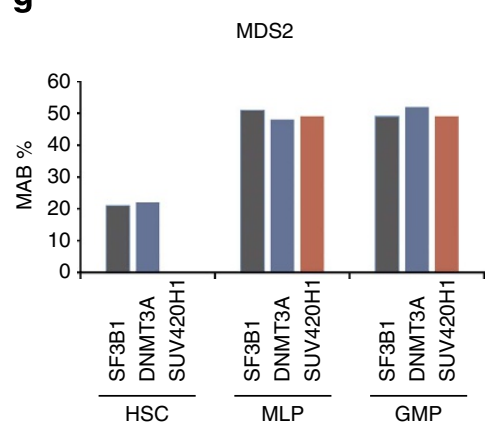

h

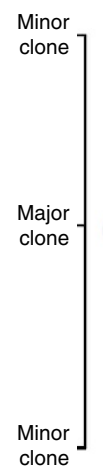

MDS2

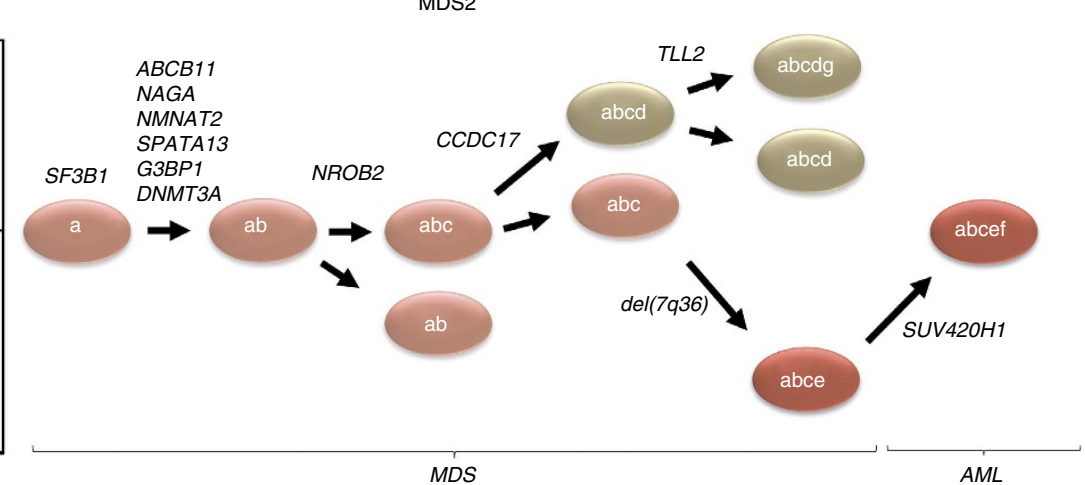

Figure 6 | Clonal evolution from MDS to AML in RCMD-RS patient with SF3B1 mutation. (a) FACS profiles of stem cell progenitors in BM MNCs in a serial sample at the MDS stage and AML stage of the disease. (b) Interphase FISH for tracking the del(7q) aberration in cells obtained at the AML stage of the disease. Two chromosome-7 aberrations, del(7q36) and del(7q22-7q36), were detected as two separate clones. Two individual cells are shown in the figure with one harbouring del(7q36) represented by a green probe and the other cell has del(7q22-7q36) represented by probes orange/green. (c) SNV profile derived from whole-exome sequencing. The shown profile depicts a heterozygous deletion (horizontal red line) of chromosome $7 q$ in patient $\mathrm{CD}_{4}{ }^{+}$bone marrow cells (AML stage, bottom) and absence of the deletion in paired control CD3 ${ }^{+}$cells (top). (d) Bar chart (FISH analysis) showing percentage of $\mathrm{CD}_{3}{ }^{+}$cells (MDS stage), CD34 ${ }^{+}$cells (AML stage), MPL (AML stage), GMP (AML stage) with del(7q) aberration. (e) Bar chart (FISH analysis) showing the percentage of $\operatorname{del}(7 q)$ aberration in LTC-derived cells from MDS-stage CD34 ${ }^{+}$cells (time point 1). (f) Mutational status of the gene mutations in sequential bone marrow samples (MDS-stage time-point 1, MDS-stage time-point 2 and AML stage). Coloured circles represent the MABs for each screened gene mutation. Black * represent absence of gene mutations in the AML-stage sample. Red * represent acquisition of additional mutation at the AML stage. (g) Mutational analysis of SF3B1, DNMT3A and SUV42OH1 in HSCs, MLPs and GMPs from the AML stage of the disease. Sequencing depth for HSCs was $>10,000$ reads. (h) Proposed sequential acquisition of genetic lesions based on analysis of sequential samples, LTC-derived cells and singlecell clonogenic assays. 


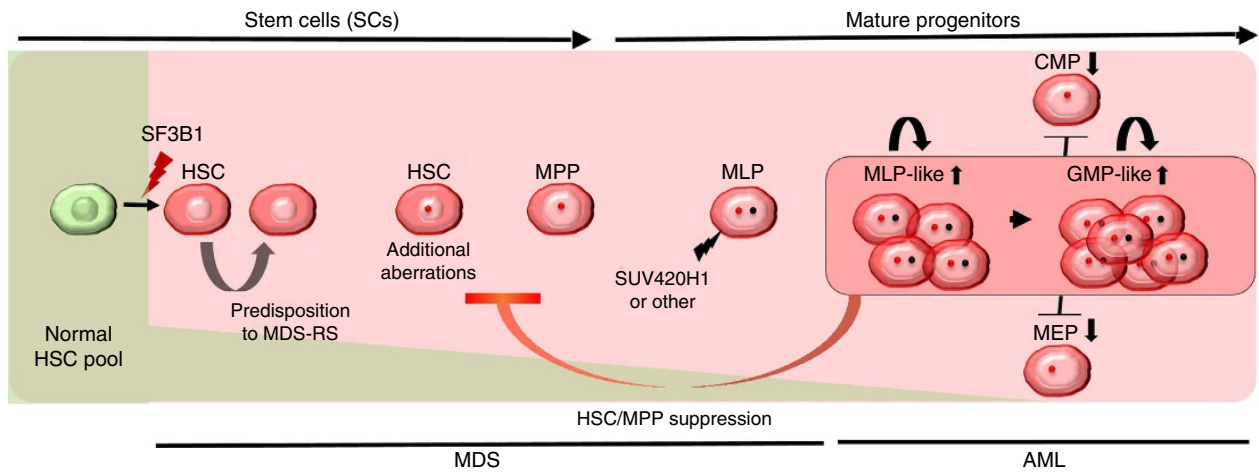

Figure 7 | Predicted model for sequential acquisition of genetic lesions in MDS to AML transformation. SF3B1 mutations are acquired in $\mathrm{CD} 34{ }^{+} \mathrm{CD} 38-\mathrm{CD} 90^{+} \mathrm{CD} 45 \mathrm{RA}-\mathrm{CD} 49 \mathrm{f}+\mathrm{HSCs}$. As HSCs replenish downstream progenitors, mutations acquired in stem cells are also propagated to mature progenitors but without conferring self-renewal potential. However, as the mature progenitors such as MLPs harbouring a mutant SF3B1 acquires a more 'potent hit' such as SUV42OH1 mutation, they confer self-renewal potential and suppress the HSC to MPP transition.

Notably, the genotypic analysis of pre- and post-transplanted cells demonstrate that in most of the cases SF3B1 mutations were maintained; however, in one case (MDS3), SF3B1 mutant clone increased from $27 \%$ in primary CD $34^{+}$cells to $47 \%$ in HEC samples. This suggests the presence of a mixed stem cell population (that is, SF3B1 mutant/wild type), which when injected into immunodeficient mice resulted in the expansion of SF3B1 mutant clones preferentially. Exclusive myeloid engraftment and the maintenance/increase in the SF3B1 clone(s) in NSG xenografts suggest that a malignant HSC clone with SF3B1 mutations either alone or in conjunction with other 'cooperating factors' has a growth advantage or a negative feedback over the cohabitating normal HSC.

Interestingly, in all mice that have detectable human engrafted cells, we could see a similar subclonal architecture compared with MDS cells injected. Nevertheless, in some cases, the engrafting SF3B1 daughter subclones represented only a small fraction of the injected cells $(<5 \%)$, implying that some SF3B1 subclones have a cell-intrinsic advantage (due to better engraftment ability, enhanced proliferation potential and/or additional factors) following xenotransplantation. Malignant clones with isolated SF3B1' mutations are not always driven away by more 'potent' mutant subclones (such as DNMT3A and/or JAK2) in NSG mice as was reflected by its MAB, which remained stable even though other subclones were fluctuating. Perhaps additional driver mutations when acquired by the SF3B1 clones confer a subtle advantage to the new daughter subclone representative of early stages in disease progression, as evidenced in MDS1 (time point 1 versus time point 2). However, when these clones acquire more aggressive mutation(s), as is the case here for MDS2, a more 'aggressive' mature progenitor clone with self-renewing capacity is generated, enabling the disease to evolve into AML. It is tempting to speculate that $S U V 420 H 1$ mutations confer a growth advantage based on the evidence that Suv420h (referred to both Suv420h1 and Suv420h2)-deficient MEFs and ES cells have been reported to have telomere elongation and epigenetic defects ${ }^{32}$. Further work needs to be done to study the role of SUV42OHI mutation(s) in haematological diseases.

It is noteworthy that a small subclone with SF3B1 mutation harbouring del(7q36) becomes a predominant clone in the sequential AML sample (MDS2), but with the additional acquisition of del(7q22). Therefore, SF3B1 mutation in isolation might only have a minor role in disease transformation or progression to AML. However, similar to del $(5 \mathrm{q})$ aberrations ${ }^{15}$, it may instead be a strong driver mutation in MDS-RS and acquires additional mutations during the course of the disease progression.
Previous studies of functional heterogeneity in human leukaemias have used NSG mice to characterize leukaemia stem cells to decipher their engraftment potential, self-renewal capacity ${ }^{33-36}$. Nevertheless, Tim Ley's group was the first to report functional heterogeneity of genetically defined subclones in $\mathrm{AML}^{14}$. Their data suggested that xenotransplantation results in a marked decrease in the subclonal complexity of the AML samples and were not able to reliably predict disease relapse. However, the combination of our WES in xenotransplanted NSG mice and serial patient sampling enabled us to compare the clonal evolution observed in mice with that of patient BM compartment in MDS1 and MDS2. The changes in the spectrum of SF3B1 mutant clones observed in the NSG mice were largely reflected in the patient's sequential BM cells where similar clonal changes were detected, although the SF3B1 clonal fluctuation observed in NSG mice for MDS1 was more remarkable as compared with MDS2.

This study demonstrates that $S F 3 B 1$ mutations can originate in rare $\mathrm{CD} 34^{+} \mathrm{CD} 38^{-} \mathrm{CD} 90^{+} \mathrm{CD} 49 \mathrm{f}^{+}$HSCs, and MDS-ICs are restricted to these rare HSC fractions in our cohort of MDS patients with ring sideroblasts. Although our data are limited to five patients, the presence of $S F 3 B 1$ mutations in $\mathrm{CD} 34^{+} \mathrm{CD} 38^{-}$ $\mathrm{CD} 90^{+} \mathrm{CD} 45 \mathrm{RA}^{-} \mathrm{CD} 49 \mathrm{f}^{+} \mathrm{HSCs}$ provides new insight into the MDS stem cells. Furthermore, our data demonstrate that SF3B1 mutations could act as an initiating event in MDS-RS disease, as has been suggested by previous studies ${ }^{3,5}$. We could not, nevertheless, exclude that in other RARS cases, mutations such as TET2 and ASXL1 (not studied here) could also act as initiating mutations. Further analysis of a larger cohort of patients, using single-cell or the clonogenic assay should address this point.

MDS2 patient progression to AML illustrates the concept of a switch from 'HSC disease' to a 'progenitor disease'. Here, we show that in some cases MDS-RS can originate from the stem cells and at a later stage of the disease de novo mutation(s) acquired by the progenitor cells can confer the self-renewal capability to the MDS progenitor cells that drives leukaemic transformation. This novel observation confirming the presence of MDS-IC at the AML stage provides new insight into the MDS/AML hierarchy. Our results also suggest that MDS-IC at the AML stage can constitute a pool of pre-leukaemic cells, providing a reservoir of cells that could be responsible for relapse. Although mutations such as SF3B1 are not driving mutations at the AML stage, they provide a genuine target for curative therapies and give the opportunity to therapeutically target at the same time the AML blasts as well as pre-leukaemic cells. 


\section{Methods}

Patients. WES data were available for 12 MDS-RS patients (Supplementary Table 1) and one congenital sideroblastic anaemia patient. In addition, one MDSRS patient with SF3B1 mutation was also included in this study. All patient samples $(n=14)$ were received from the King's College London Haemato-Oncology Tissue Bank. All patients had provided written informed consent for the samples to be used for all the experiments in accordance to the King's College London HaematoOncology Tissue Bank research ethics protocol (08/H0906/94). Demographic and clinical characteristics of the studied patients are detailed in Supplementary Table 1. All patients were risk stratified according to IPSS categories. The clinical variables for all patients were ascertained at the time of sample collection. Sequential (post AML transformation) samples from MDS2 was also available (Supplementary Tables 1 and 4).

BM aspirates from 6/12 MDS-RS (MDS1, MDS2, MDS3, MDS4, MDS6 and MDS14) all containing the $S F 3 B 1$ mutations (K700E, $n=2 ; \mathrm{H} 662 \mathrm{Q}, n=3)$ and one congenital sideroblastic anaemia patient (MDS5, wild-type SF3B1) were selected for in vivo, in vitro experiments and/or haematopoietic cell separation (Supplementary Tables 1 and 4).

Xenotransplant assays. MNCs were isolated from the BM cells by centrifugation using Ficoll-Paque PLUS (GE Healthcare Life Sciences, Buckinghamshire, UK). The cells were processed within $24 \mathrm{~h}$ following collection using an Easysep Human CD34-positive selection kit and Easysep magnet (StemCell Technologies, Vancouver, Canada) according to the manufacturer's instructions to enrich CD34 ${ }^{+}$ cells from patient samples. Mesenchymal stem cells were isolated from the CD34 fraction during the $\mathrm{CD} 34^{+}$cell selection. Cells were seeded at a concentration of $1.10^{6} / \mathrm{cm}^{2}$, in DMEM low glucose (Life Technologies, Paisley, UK) supplemented with fetal bovine serum (Mesenchymal Stem Cell Qualified, Life Technologies). Culture media was replaced 3 days later, and cells were frozen at passage-2 maximum.

NSG mice were a kind gift of Dr Leonard Shultz (The Jackson Laboratory). All animal experiments were performed in accordance to Home Office and CRUK guidelines. Female NSG mice used in this study were 8 to 12 weeks old at the start of the experiments. Before transplantation, mice received a sub-lethal dose of radiation ( $375 \mathrm{cGy}$ ) from a caesium-137 source. Direct intra-BM injection was performed in the tibia with $0.65 \times 10^{5}$ to $2 \times 10^{5} \mathrm{BM} \mathrm{CD} 34^{+}$cells from patients and healthy donors.

Engraftment was assessed over time by intra-tibia aspiration under isoflurane anaesthesia, and the $\mathrm{BM}$ was immunophenotyped by the presence of $\mathrm{mCD} 45^{-}$, $\mathrm{hCD}^{+} 5^{+}, \mathrm{hCD}_{3}{ }^{+}, \mathrm{hCD}_{19}{ }^{-}$and $\mathrm{hCD} 3^{-}$(using anti-mouse and anti-human antibodies from BD Biosciences, Oxford, UK) cell populations. After 18-20 weeks, mice experiments were terminated and cells were harvested from pooled BM (femurs, tibias, pelvis and/or spine). Live cells were stained and sorted on the hCD $45^{+}$phenotype using FACS Aria SORP (BD Biosciences). Sorted cells were washed in PBS and harvested to later perform genomic analysis.

CD45 Immunohistochemistry. Femurs were collected from engrafted and nonengrafted mice, washed in PBS, fixed overnight at $4{ }^{\circ} \mathrm{C}$ in $4 \%$ paraformaldehyde (methanol free). Samples were washed in PBS once before being washed in water. Bones were incubated $2 \mathrm{~h}$ on ice in $8 \% \mathrm{HCl}$ solution for decalcification. Samples were rinsed with water and stored in PBS before immunostaining.

Tissues were fixed in $10 \%$ neutral buffered formalin overnight and processed for paraffin embedding and sectioned at $4 \mathrm{M}$. Sections were microwaved for $15 \mathrm{~min}$ in $0.01 \mathrm{M}$ sodium citrate buffer $\mathrm{pH} 6$ for antigen retrieval. Sections were blocked with $1.6 \% \mathrm{H}_{2} \mathrm{O}_{2} / \mathrm{PBS}$ and then $10 \%$ normal horse serum/1\% BSA and stained for $1 \mathrm{~h}$ with mouse anti-CD45 (Dako M0701, Cambridgeshire, UK) at 1/200 dilution. Secondary antibody was used with biotinylated horse anti-mouse IgG (Vector Labs, Peterborough, UK), followed by $\mathrm{ABC}$ reagent (Vector Labs), developed with $\mathrm{DAB}$ (Vector Labs) and counterstained with haematoxylin. Pictures were taken using an inverted microscope and analysed with NIS-Elements software from Nikon.

CD45 immunohistochemistry. Femurs were collected from engrafted and nonengrafted mice, washed in PBS, fixed overnight at $4{ }^{\circ} \mathrm{C}$ in $4 \%$ paraformaldehyde (methanol free). Samples were washed in PBS once before being washed in water. Bones were incubated $2 \mathrm{~h}$ on ice in $8 \% \mathrm{HCl}$ solution for decalcification. Samples were rinsed with water and stored in PBS before immunostaining.

Tissues were fixed in $10 \%$ neutral buffered formalin overnight and processed for paraffin embedding and sectioned at $4 \mathrm{M}$. Sections were microwaved for $15 \mathrm{~min}$ in $0.01 \mathrm{M}$ sodium citrate buffer $\mathrm{pH} 6$ for antigen retrieval. Sections were blocked with $1.6 \% \mathrm{H}_{2} \mathrm{O}_{2} / \mathrm{PBS}$ and then $10 \%$ normal horse serum $/ 1 \% \mathrm{BSA}$ and stained for $1 \mathrm{~h}$ with mouse anti-CD45 (Dako M0701) at 1/200 dilution. Secondary antibody was used with biotinylated horse anti-mouse IgG (Vector Labs), followed by ABC reagent (Vector Labs), developed with DAB (Vector Labs) and counterstained with haematoxylin. Pictures were taken using an inverted microscope and analysed with NIS-Elements software from Nikon.

Isolation of HSCs and progenitors. BM MNCs were separated from total BM cells by density-gradient sedimentation with Histopaque-1077 (Sigma-Aldrich Co. LLC). Up to $1 \times 10^{8}$ MNCs were resuspended in $350 \mu \mathrm{l}$ of auto-MACS running buffer and subsequently used for immunomagnetic enrichment of $\mathrm{CD} 34^{+}$cells using MACS. CD34 ${ }^{+}$cells were enriched by positive selection as per the manufacturer's instructions (Miltenyi Biotec Ltd, CD34 MicroBead Kit Human). CD34 ${ }^{+}$ cells were kept on ice for subsequent use and $\mathrm{CD} 34^{-}$cells were stored as viable cells in Synth-A-Freeze media (Life Technologies) at $-80^{\circ} \mathrm{C}$.

Up to $1 \times 10^{6} \mathrm{CD}_{4}{ }^{+}$cells were stained with the following antibodies: $5 \mu \mathrm{l}$ of CD45 (APC-eFluor 780, clone HI30), $1 \mu \mathrm{l}$ of CD49f (FITC, clone GoH3), $5 \mu \mathrm{l}$ of CD34 (PE-Cy7, clone 4H11), $5 \mu \mathrm{l}$ of CD38 (APC, clone HIT2), $5 \mu \mathrm{CD} 135$ (PE, clone BV10A4H2) (eBioscience), $5 \mu \mathrm{l}$ of CD45RA (BV 510,clone HI100), $2 \mu \mathrm{l}$ of CD90 (Alexa Fluor 700, clone5E10) (Biolegend UK Ltd) and a lineage-specific antibody cocktail ( $1 \mu \mathrm{l}$ of CD2 biotin, $1 \mu \mathrm{l}$ of CD3 biotin, $1 \mu \mathrm{l}$ of CD14 biotin, $1 \mu \mathrm{l}$ of CD16 biotin, $1 \mu \mathrm{l}$ of CD19 biotin, $1 \mu \mathrm{l}$ of CD24 biotin, $1 \mu \mathrm{l}$ of CD56 biotin, $1 \mu \mathrm{l}$ of CD235a biotin; together with $5 \mu \mathrm{l}$ of Streptavidin eFluor 450, all by eBiosciences and CD66b (V450) (BD Biosciences). Propidium iodide was added to the cell suspension before sorting to exclude dead cells. Cells were sorted on a BD FACS Aria SORP (San Jose, CA) operating in a four-way purity sort mode, and collected into $1.5-\mathrm{ml}$ microfuge tubes. Our cell populations of interest were defined by hierarchical gating, which was largely similar to the one used by Notta et al. ${ }^{23}$, HSCs as $\mathrm{Lin}^{-} \mathrm{CD} 45^{+} \mathrm{CD} 34^{+} \mathrm{CD} 38^{-} \mathrm{CD}^{-} 5 \mathrm{RA}^{-} \mathrm{CD} 90^{+} \mathrm{CD} 49 \mathrm{f}^{+}$, MPPs as $\mathrm{Lin}^{-} \mathrm{CD} 45^{+} \mathrm{CD} 34^{+} \mathrm{CD} 38^{-}{ }^{-} \mathrm{CD} 45 \mathrm{RA}^{-}{ }^{-} \mathrm{CD} 90^{-} \mathrm{CD} 49 \mathrm{f}^{-}$, GMPs as $\mathrm{Lin}^{-} \mathrm{CD}^{-} 5^{+} \mathrm{CD} 4^{+}{ }^{+} \mathrm{CD} 38^{+}{ }^{+} \mathrm{CD} 135^{+}{ }^{+} \mathrm{CD} 45 \mathrm{RA}{ }^{+}$and MEPs as $\mathrm{Lin}^{-} \mathrm{CD} 45^{+}$ $\mathrm{CD} 34^{+} \mathrm{CD} 38^{+} \mathrm{CD} 135^{-} \mathrm{CD} 45 \mathrm{RA}^{-}$(Supplementary Fig. 1). Moreover, in the case of the AML sample, CD123 antibody (PE, clone: $6 \mathrm{H} 6$, eBioscience) was used instead of CD135 to define the CMP, MEP and GMP compartments.

After sorting, the purity of MPPs, GMPs and MEPs were checked and a sorting purity of $\geq 97 \%$ was routinely obtained. Cells were spun down after sorting, supernatant was discarded and cells were either stored at $-80^{\circ} \mathrm{C}$ and/or resuspended in Karnoy's solution to later perform FISH analysis.

Whole-genome amplification. WGA of cells was performed with the GenomePlex Single Cell Whole Genome Amplification kit (WGA4, Sigma-Aldrich Co, LLC) and/or GenomePlex SeqPlex XE kit (SEQXE, Sigma-Aldrich Co., LLC). WGA amplification was performed according to the instructions of the manufacturer, along with a no-cell reaction as a negative control and a reaction of human tissue genomic DNA (gDNA) as a positive control.

For patient samples where only one mouse was used in in vivo experiments, two independent amplification experiments were performed using two different amplification kits (that is, WGA4 and SEQXE). In addition, all patients samples, the HSCs and MPPs were also amplified by two different amplification kits (WGA4 and SEQXE), while the GMPs and MEPs were amplified by using the SEQXE WGA kit.

WES and data analysis. Thirteen MDS-RS patients were selected for WES, using DNA from $\mathrm{CD}_{3}{ }^{+}$cells (primary BM sample) in all cases and paired constitutional DNA from 11 cases (skin, $n=7 ; \mathrm{CD}^{+} n=4$ ). One additional MDS-RS patient was subjected to targeted mutational analysis. The WES data have been deposited in the NCBI Sequence Read Archive under accession code XXX.

$\mathrm{CD} 45^{+} \mathrm{CD} 33^{+}$cells derived from mice (human engrafted cells) from four of these patients (MDS1, MDS2, MDS3 and MDS4) were also subjected to WES. WES was also performed on three LTC-derived samples from three patients (for more details, see Supplementary Table 4). For cases where WGA was performed using two independent WGA kits, both WGA samples were independently processed for WES.

gDNA (non-WGA, 100-500 ng) or WGA DNA (500 ng) was processed for exome sequencing (Agilent V4) and sequenced on the Illumina HiSeq2000 (Paired end V3 chemistry) according to manufacturer's instructions. Base calling was generated by the Illumina RTA software. Demultiplexing and conversion of basecalls to fastq was performed by Casava version 1.8.2, filtering out poor-quality reads fastq files concatenated. Alignment, realignment and recalibration was performed using Burrows-Wheelers aligner ${ }^{37}$ and GATK $^{38}$, respectively, according to the Broad Institute best practices for individual samples. VarScan 1.3.4 (refs 39,40 ) was subsequently used to call somatic variants on pileup files (Samtools) ${ }^{41}$ for paired tumour and normal (skin or $\mathrm{CD}^{+}{ }^{+} \mathrm{T}$ cells) tissue. Reads with an alignment score of $<10$, base quality score $<15$ and fold strand bias $>10$ were excluded. Pindel ${ }^{42}$ was also used to screen for more computationally challenging Indel mutations at specific loci, such as for FLT3 ITD. All resulting variants were passed through ANNOVAR ${ }^{43}$ using refseq annotation, and variants deemed to cause protein changes and not found in dbSNP135, esp5400 and 6500 and 1,000 genomes databases at $>0.001$ population allele frequency were passed. Genomic duplicated regions were also filtered out unless associated with known mutations. Somatic mutations were subsequently passed when the somatic $P$ value (VarScan, Fisher's exact $T$-test) was $<0.01$, had $\geq 3$ reads supporting mutation, were present in paired normal skin tissue at $<20 \%$ (or for paired $t$-cells $<50 \%$ ) at $<20 \%$ of the paired tumour tissue and had an allele burden of $>5 \%$. For isolated HEC CD $45^{+} \mathrm{CD}_{3}{ }^{+}$cells, candidate mutations were only passed if they were present in $>1$ HEC sample or found concurrently in one or more HEC experiment and LTC-derived sample or primary CD $34^{+}$cells for the same patient. Where only WGA-derived data from HEC were available for one engraftment experiment, or a combination of WGA and non-WGA data from more than one engraftment experiment, mutations were only passed when they were found concurrently in 
either: (1) WGA HEC and non-WGA HEC-independent experiments; (2) repeat WGA DNA samples from the same engraftment experiments or; (3) WGA HEC experiments and LTC-derived sample, CD34 ${ }^{+}$or HEC experiments derived from non-WGA DNA. For WGA-derived HEC, a stand-alone analysis was also performed for individual experiments. For this, additional false-positive filters were applied to remove additional WGA-derived artefacts with positional and readlength distribution bias, as well as variant and mapping quality biases. Variants with homopolymer runs of five or more flanking the mutation were also filtered out. WGA-derived variants from control WGA experiments were also removed. For MDS3, only variants found in at least two of the three animal experiments from WGA-derived data were included in the final list due to increased artefactual events. Stand-alone analysis of non-WGA HEC cells were likewise performed but only using filters up to step 7 above.

Mapping loss of heterozygozity across chromosomes by WES. Loss of heterozygosity was mapped from WES data according to the following criteria: variants were called as described above. Variants found in the 1,000 genome project database with a population allele frequency $<0.001$ were selected and filtered out for known genomic duplicated regions, read depth $<20$ and somatic status according to VarScan. The filtered set of variants was then plotted graphically for the required chromosome.

Exome coverage. Exome coverage was calculated utilizing GATK Depth Of Coverage script against intervals that the Agilent V4 Sureselect kit capture probes were based on. For non-WGA sample, mean base coverage across the targeted region ranged from $\times 77$ to 115 and for $>98 \%$ of the exome base coverage was $>10$ reads in all cases, based on 9-13 gigabase of sequence data. For the same amount of sequence data on average, WGA-derived exome data yielded an average base coverage of $\times 55-75$ and $65-83 \%$ (average $74 \%$ ) of exome covered $\geq 10$ reads.

Sequencing validation experiments. All somatic mutations were confirmed independently by sequencing from three independent PCR reactions from all experiments $\left(\mathrm{CD} 34^{+}\right.$day-0 samples, HEC from mice, LTC and CFC), which enabled us to quantify the MAB more accurately in all cases.

Primers for candidate gene variants were designed by using Primer 3 program ${ }^{44}$ with default settings. Using relevant candidate gene-specific primers and GoTaq Hot Start Colorless Master Mix (Promega), PCR amplification was performed on all primary $\mathrm{CD} 34^{+}$samples, HEC samples, LTC-derived cells and pooled CFC by following manufacturer's protocol. The acquired nature of the mutations was also confirmed by their absence in paired constitutional DNA. In the case of WGAderived candidate mutations, validation was also confirmed in at least one gDNA (non-WGA) sample from that particular patient.

In the case of WGA-derived candidate mutations, validation was also confirmed in at least one gDNA (non-WGA) sample from that particular patient. The degree of quantitative accuracy of the allele burdens for WGA DNA was validated by performing WES on paired gDNA (non-WGA) and WGA DNA from the samples (Supplementary Fig. 3). For this, we observed a similar distribution of known single-nucleotide polymorphisms across the exome between the gDNA (nonWGA) and WGA DNA. To validate this further, we also performed mutational screening for 10 genes on paired gDNA (non-WGA) and WGA DNA from two (MDS1 and MDS2) independent patients (Supplementary Fig. 3). MAB for specific mutations was also shown to be comparable between gDNA (non-WGA) and WGA DNA in targeted amplicon sequencing in these control experiment.

For MDS1, all the mutations including SF3B1 were confirmed in all mice experiments using gDNA (non-WGA) DNA. In addition, all mutations were also confirmed in LTC (non-WGA) and CFC (non-WGA) samples. For MDS2, all the mutations were confirmed in amplified DNA from mice; however, $S F 3 B 1$ and four of the additional mutations were confirmed on gDNA (non-WGA) from mice. In addition, all mutations were also confirmed in LTC (non-WGA) and CFC (nonWGA) samples. For MDS3, all the mutations including SF3B1 identified in two animals from amplified DNA were also found in one gDNA (non-WGA) from mice. In addition, all mutations were also confirmed in LTC (non-WGA) and CFC (non-WGA) samples. For MDS4, SF3B1 and one additional mutation was confirmed in mouse gDNA (non-WGA).

Furthermore, all somatic mutations were confirmed independently by sequencing from three independent PCR reactions from all mice experiments, which enabled us to quantify the MAB more accurately in all cases. The degree of quantitative accuracy of the allele burdens for WGA DNA was validated by performing WES on paired gDNA (non-WGA) and WGA DNA from the samples (Supplementary Fig. 2). For this, we observed a similar distribution of known single-nucleotide polymorphisms across the exome between the gDNA (nonWGA) and WGA DNA. To validate this further, we also performed mutational screening for 10 genes on paired gDNA (non-WGA) and WGA DNA from 2 (MDS1 and MDS2) independent patients (Supplementary Fig. 2). MAB for specific mutations was also shown to be comparable between gDNA (non-WGA) and WGA DNA in targeted amplicon sequencing in these control experiments.

Here, PCR amplicons were normalized, mixed in batches and converted into Illumina sequencing libraries utilizing transposon-based Illumina-Nextera technology (Illumina), designated nextera-amplicon libraries here. These libraries were sequenced on the Illumina MiSeq utilizing version 2 chemistry, 150-250 paired-end reads and dual indexes allowing multiplexing of 96 samples independent of sequencing experiments utilizing the nextera-amplicon methodology.

Primers used for validation. Target genes oligo sequence $\left(5^{\prime}\right.$ to $\left.3^{\prime}\right)$

SF3B1_14f CCAACTCATGACTGTCCTTTCTT

SF3B1_14r GGGCAACATAGTAAGACCCTGT

SF3B1_15f TTGGGGCATAGTTAAAACCTG

SF3B1_15r TTCAAGAAAGCAGCCAAACC

JAK2_E14f TCCTCATCTATAGTCATGCTGAAA

JAK2_E14r CTGACACCTAGCTGTGATCCTG

DNMT3A_E8r TCTTGCCTCATTCAGATGGA

DNMT3A_E8r CCTGGGATCAAGAACCTTCC

DNMT3A_E15f ACCAGGGCTGAGAGTCTCCT

DNMT3A_E15r AGGCTCCTAGACCCACACAC

FBLIM1_f CGAGAAGGGTTTGTGCACTG

FBLIM1_r TCCCAGGTTCACGCCATT

HIST1H2AC_f CGCGACAACAAGAAGACTCG

HIST1H2AC r GCAGTGAGGTTAGCTCTTCC

HSPA12A_f CCAGTGTGCCCTCAAAAGTC

HSPA12A_r CTTATGCAGTTCGGGGACAC

RETNLB_f AAGAATGGGCAAGGGGTCTC RETNLB_r ATGGGGAGGATAGCTCATGG

SMC6_f TGGAACATTAGGGCCAAATCT

SMC6_r GCCAAGTTTTGAGGACTCCA

SP3_f AGCCAGACTGACTTGTTCTTTG

SP3 r CTGGAGAACGCCCTTTTGTT

TH_f CACGGATGTGTAGCAAAACG

TH_r CCTGCTTTTGCTCCCTAAGA

NAGA_f CGTAGTCAGCCAGGAAAGGA

NAGA_r GCTCAGCAAGGTCATGGTTT

NMNAT2_f CTCTCTCACTCCTCCAGCTG

NMNAT2_r TGTGGAAGAATGGAAGCGGA

NR0B2_f GCAAAAGCATGTCCCCAAGA

NR0B2_r CAGTCTTGTCCTTTGGTGGC

SPATA13_f CGCCGTGTGTGAGATTCTC

SPATA13 r CAGCCTCAGTCACATTGCTC

TLL2_f CCCCTCAATACAGTCCCCAA

TLL2_r AGGCTCTATTGCTCCTGGTG

G3BP1_f AGTCAGTGCCATGATTTTACCA

G3BP1_r ACAATGCAAGTTCTTATGCAGC

ABCB11_f AACAGACCAGCACTCACCTT

ABCB11_r TCATAAAACAGAGCAACAACCAG

CCDC17_f CTTTCCAGAACTGCTCAGCG

CCDC17 r GAGACCCGACTAAGCGGATC

ME1_f TACCTTGGCTTCCGAAACAC

ME1_r ACAGGCATGAACCACTGTGC

CAMTA1_f GTTCTCTGCAGGGAGGAGTG

CAMTA1_r CTGCCCTAAATCGTGCTCTC

PTPDC1_f CGCAAATTAGAGGATCACAACA

PTPDC1_r CCCTTCCTCCAGAAATGTGT

MYH14_f AAGACCCGGATATGGGAAGT

MYH14_r TCCTCCCTCAACAGATCCAC

Colony-forming cell. Five hundred cells from the $\mathrm{BM} \mathrm{CD} 34^{+}$-enriched fraction were plated in triplicate in $0.5 \mathrm{ml}$ of MethoCult H4434 (StemCell Technologies). At day 14 of culture, the numbers of colonies were scored. Single-cell colony images were acquired with a Zeiss AxioVert 40 CFL microscope using $\times 5$ lens and connected to Canon PowerShot A640 digital camera. Cells were picked, harvested and washed twice with PBS. All CFC colonies were screened for SF3B1 and additional patient-specific mutations.

LTC assay. LTC assays were performed by plating $10^{3} \mathrm{CD} 34^{+} \mathrm{BM}$ cells in quadruplets on irradiated MS-5 murine stromal cells and cultured in Myelocult $\mathrm{H} 5100$ (StemCell Technologies) in the presence of cytokines $\left(20 \mathrm{ng} \mathrm{ml}^{-1} \mathrm{G}-\mathrm{CSF}\right.$, $20 \mathrm{ng} \mathrm{ml}^{-1} \mathrm{IL}-3$ and $20 \mathrm{ng} \mathrm{ml}^{-1}$ TPO from PeproTech, London, UK). After 5 weeks, live cells were stained and sorted on the human $\mathrm{CD} 45^{+}$phenotype using the FACS Aria SORP (BD Biosciences). Sorted cells were washed in PBS, harvested and stored as cell pellet and/or resuspended in Karnoy's solution to later perform FISH and sequencing analysis.

Genotyping of single-cell clones and/or colonies. CFU-GM and BFU-E colonies were harvested for four patients (MDS1, MDS2, MDS3 and MDS4) at day 14, and washed twice with PBS. All colonies were subjected to WGA (single-cell WGA, Sigma-Aldrich). All CFC colonies were screened for SF3B1 and additional patientspecific point mutations. Amplicon libraries were amplified through PCR using the primers specific to gene mutations and subjected to Nextra-Illumina sequencing. Gene mutation for each single colony was called when $>30 \%$ of the sequencing reads were carrying a mutant allele. 
Amplicon sequencing for cell fractions. WGA-derived DNA from cell fractions (HSCs, MPPs, GMPs and MEPs) were subjected to sequencing. Candidate gene mutations from the WES data were followed-up specifically. These mutations were also screened in mesenchymal stem cells $(n=4)$ derived from the concurrent patients. Furthermore, all somatic mutations were confirmed independently by sequencing from $\geq 2$ independent PCR reactions. Sequencing was performed using Nextra Technology (Illumina) and MiSeq platform (Illumina) as described previously.

MAB is presented as an average of at least two independent Illumina-Nextera validation experiments combined with data from exome sequencing where available. In this way, the variation expected from sampling error when working with small progenitor cell populations was minimized. The average sequencing coverage across all amplicons was $\geq 800 \times$. This coverage enabled us to reliably detect mutant clones down to $\geq 1 \% \mathrm{MAB}$, defined as the proportion of sequence reads containing the mutation.

SF3B1 gene mutation were analysed in haematopoietic cell fractions (HSCs, MPPs, GMPs and MEPs) by PCRs directly on $30-50 \%$ of the cell fraction with subsequent sequencing. For one additional patient (MDS6), which was not used for in vivo and in vitro experiments, SF3B1 mutation status was assessed in cell fractions in the same manner.

Fluorescence in situ hybridization. Cells were pelleted, washed twice in PBS and then resuspended in 0.5 to $1 \mathrm{ml}$ of Karnoy's solution (3:1 methanol/acetic acid glacial, purchased from Sigma-Aldrich and Fisher Chemical, respectively) and then stored at $4{ }^{\circ} \mathrm{C}$ before hybridization. For interphase FISH, fixed cells were centrifuged and an appropriate amount of supernatant was removed. Pelleted cells were then transferred onto the slides and hybridized with a XL 7q22/7q36 (Metasystems) locus-specific probe, which detects deletions in the long arm of chromosome 7 (Supplementary Fig. 6). The orange-labelled probe targets a specific region at $7 \mathrm{q} 22$ and the green-labelled probe binds specifically to $7 \mathrm{q} 36$. In addition, a blue (aqua)-labelled probe, which hybridizes to the centromere of chromosome 7 functions as a reference probe. Fluorescence images were obtained with the help of fluorescence microscopy. In all, 200-600 interphase cells were analysed per sample.

Statistical analysis. Prism Version 6 software (GraphPad) was used for statistical analysis. Data are presented as the mean \pm s.e.m. Statistical analysis was performed using the two-tailed Student's $t$-test for comparison of two groups to determine the level of significance.

\section{References}

1. Mufti, G. J. et al. Diagnosis and classification of myelodysplastic syndrome: International Working Group on Morphology of myelodysplastic syndrome (IWGM-MDS) consensus proposals for the definition and enumeration of myeloblasts and ring sideroblasts. Haematologica 93, 1712-1717 (2008).

2. Tefferi, A. \& Vardiman, J. W. Myelodysplastic syndromes. N. Engl. J. Med. 361, 1872-1885 (2009).

3. Yoshida, K. et al. Frequent pathway mutations of splicing machinery in myelodysplasia. Nature 478, 64-69 (2011).

4. Mian, S. A. et al. Spliceosome mutations exhibit specific associations with epigenetic modifiers and proto-oncogenes mutated in myelodysplastic syndrome. Haematologica 98, 1058-1066 (2013).

5. Papaemmanuil, E. et al. Somatic SF3B1 mutation in myelodysplasia with ring sideroblasts. N. Engl. J. Med. 365, 1384-1395 (2011).

6. Damm, F. et al. SF3B1 mutations in myelodysplastic syndromes: clinical associations and prognostic implications. Leukemia 26, 1137-1140 (2012).

7. Visconte, V. et al. SF3B1 haploinsufficiency leads to formation of ring sideroblasts in myelodysplastic syndromes. Blood 120, 3173-3186 (2012).

8. Haferlach, T. et al. Landscape of genetic lesions in 944 patients with myelodysplastic syndromes. Leukemia 28, 241-247 (2014).

9. Isono, K., Mizutani-Koseki, Y., Komori, T., Schmidt-Zachmann, M. S. \& Koseki, H. Mammalian polycomb-mediated repression of Hox genes requires the essential spliceosomal protein Sf3b1. Genes Dev. 19, 536-541 (2005)

10. Greaves, M. \& Maley, C. C. Clonal evolution in cancer. Nature 481, 306-313 (2012)

11. Shackleton, M., Quintana, E., Fearon, E. R. \& Morrison, S. J. Heterogeneity in cancer: cancer stem cells versus clonal evolution. Cell 138, 822-829 (2009).

12. Swanton, C. Intratumor heterogeneity: evolution through space and time. Cancer Res. 72, 4875-4882 (2012).

13. Anderson, K. et al. Genetic variegation of clonal architecture and propagating cells in leukaemia. Nature 469, 356-361 (2011).

14. Klco, J. M. et al. Functional heterogeneity of genetically defined subclones in acute myeloid leukemia. Cancer Cell. 25, 379-392 (2014).

15. Woll, P. S. et al. Myelodysplastic syndromes are propagated by rare and distinct human cancer stem cells in vivo. Cancer Cell. 25, 794-808 (2014).

16. Makishima, H. et al. Mutations in the spliceosome machinery, a novel and ubiquitous pathway in leukemogenesis. Blood 119, 3203-3210 (2012).
17. Kaneko, K. et al. Identification of a novel erythroid-specific enhancer for the ALAS2 gene and its loss-of-function mutation which is associated with congenital sideroblastic anemia. Haematologica 99, 252-261 (2014).

18. Cotter, P. D. et al. Four new mutations in the erythroid-specific 5aminolevulinate synthase (ALAS2) gene causing X-linked sideroblastic anemia increased pyridoxine responsiveness after removal of iron overload by phlebotomy and coinheritance of hereditary hemochromatosis. Blood 93, 1757-1769 (1999).

19. Camaschella, C. Hereditary sideroblastic anemias: pathophysiology, diagnosis, and treatment. Semin Hematol. 46, 371-377 (2009).

20. Papaemmanuil, E. et al. Clinical and biological implications of driver mutations in myelodysplastic syndromes. Blood 122, 3616-3627 (2013).

21. Bejar, R. et al. Validation of a prognostic model and the impact of mutations in patients with lower-risk myelodysplastic syndromes. J. Clin. Oncol. 30, 3376-3382 (2012).

22. Pang, W. W. et al. Hematopoietic stem cell and progenitor cell mechanisms in myelodysplastic syndromes. Proc. Natl Acad. Sci. USA 110, 3011-3016 (2013)

23. Notta, F. et al. Isolation of single human hematopoietic stem cells capable of long-term multilineage engraftment. Science 333, 218-221 (2011).

24. Bonnet, D. \& Dick, J. E. Human acute myeloid leukemia is organized as a hierarchy that originates from a primitive hematopoietic cell. Nat. Med. 3, 730-737 (1997).

25. Medyouf, H. et al. Myelodysplastic cells in patients reprogram mesenchymal stromal cells to establish a transplantable stem cell niche disease unit. Cell Stem Cell 14, 824-837 (2014).

26. Fraga, M. F. et al. Loss of acetylation at Lys 16 and trimethylation at Lys 20 of histone H4 is a common hallmark of human cancer. Nat. Genet. 37, 391-400 (2005).

27. Tryndyak, V. P., Kovalchuk, O. \& Pogribny, I. P. Loss of DNA methylation and histone $\mathrm{H} 4$ lysine 20 trimethylation in human breast cancer cells is associated with aberrant expression of DNA methyltransferase 1, Suv4-20h2 histone methyltransferase and methyl-binding proteins. Cancer Biol. Ther. 5, 65-70 (2006).

28. Ding, L. et al. Clonal evolution in relapsed acute myeloid leukaemia revealed by whole-genome sequencing. Nature 481, 506-510 (2012).

29. Mardis, E. R. et al. Recurring mutations found by sequencing an acute myeloid leukemia genome. N. Engl. J. Med. 361, 1058-1066 (2009).

30. Welch, J. S. et al. The origin and evolution of mutations in acute myeloid leukemia. Cell 150, 264-278 (2012).

31. Walter, M. J. et al. Recurrent DNMT3A mutations in patients with myelodysplastic syndromes. Leukemia 25, 1153-1158 (2011).

32. Benetti, R. et al. Suv4-20h deficiency results in telomere elongation and derepression of telomere recombination. J. Cell Biol. 178, 925-936 (2007)

33. Taussig, D. C. et al. Leukemia-initiating cells from some acute myeloid leukemia patients with mutated nucleophosmin reside in the CD34(-) fraction. Blood 115, 1976-1984 (2010).

34. Taussig, D. C. et al. Anti-CD38 antibody-mediated clearance of human repopulating cells masks the heterogeneity of leukemia-initiating cells. Blood 112, 568-575 (2008).

35. Goardon, N. et al. Coexistence of LMPP-like and GMP-like leukemia stem cells in acute myeloid leukemia. Cancer Cell 19, 138-152 (2011).

36. Eppert, B. L., Wortham, B. W., Flury, J. L. \& Borchers, M. T. Functional characterization of $\mathrm{T}$ cell populations in a mouse model of chronic obstructive pulmonary disease. J. Immunol. 190, 1331-1340 (2013).

37. Li, H. \& Durbin, R. Fast and accurate short read alignment with BurrowsWheeler transform. Bioinformatics 25, 1754-1760 (2009).

38. McKenna, A. et al. The Genome Analysis Toolkit: a MapReduce framework for analyzing next-generation DNA sequencing data. Genome Res. 20, 1297-1303 (2010).

39. Koboldt, D. C. et al. VarScan 2: somatic mutation and copy number alteration discovery in cancer by exome sequencing. Genome Res. 22, 568-576 (2012).

40. Koboldt, D. C. et al. VarScan: variant detection in massively parallel sequencing of individual and pooled samples. Bioinformatics 25, 2283-2285 (2009).

41. Li, H. et al. The Sequence alignment/map format and SAMtools. Bioinformatics 25, 2078-2079 (2009).

42. Ye, K., Schulz, M. H., Long, Q., Apweiler, R. \& Ning, Z. Pindel: a pattern growth approach to detect break points of large deletions and medium sized insertions from paired-end short reads. Bioinformatics 25, 2865-2871 (2009).

43. Wang, K., Li, M. \& Hakonarson, H. ANNOVAR: functional annotation of genetic variants from high-throughput sequencing data. Nucleic Acids Res. 38, e164 (2010).

44. Koressaar, T. \& Remm, M. Enhancements and modifications of primer design program Primer3. Bioinformatics 23, 1289-1291 (2007).

\section{Acknowledgements}

We would like to acknowledge the leukaemia and Lymphoma Research Fund (UK) for supporting S.A.M. and K.R.-P. We also thank King's College London and Kings College Hospital NHS trust for funding the King's College Hemato-Oncology Tissue Bank and 
CRUK core funding to D.B. We acknowledge Nigel B. Westwood, Rajani Chelliah and Swaibu Mugambwa for assisting with sample processing, tissue separation and clinical data. We thank Biological Resource Units, the Bioinformatics and Biostatistics Services and the Flow Cytometry core facility members at LRI.

\section{Author contributions}

S.A.M. and K.R.-P. contributed equally and were involved with all aspects of the study's design, execution, analysis and manuscript preparation. G.J.M. and D.B. contributed equally to design, analysis and manuscript preparation, as well as providing project leadership. A.E.S. contributed to design, data analysis and manuscript preparation. A.G.K. contributed to clinical data. T.S., A.K. and I.P. contributed to experiments and analysis. All authors reviewed the manuscript during its preparation.

\section{Additional information}

Accession codes: Data available on EBML Nucleotide Sequence Database (http:// www.ebi.ac.uk/ena) with the accession number: PRJEB12004.
Supplementary Information accompanies this paper at http://www.nature.com/ naturecommunications

Competing financial interests: The authors declare no competing financial interests.

Reprints and permission information is available online at http://npg.nature.com/ reprintsandpermissions/

How to cite this article: Mian, S. A. et al. SF3B1 mutant MDS-initiating cells may arise from the haematopoietic stem cell compartment. Nat. Commun. 6:10004 doi: $10.1038 /$ ncomms10004 (2015).

(c) (i) This work is licensed under a Creative Commons Attribution 4.0 International License. The images or other third party material in this article are included in the article's Creative Commons license, unless indicated otherwise in the credit line; if the material is not included under the Creative Commons license, users will need to obtain permission from the license holder to reproduce the material. To view a copy of this license, visit http://creativecommons.org/licenses/by/4.0/ 